\title{
Absorbing Boundary Conditions in Quantum Relativistic Mechanics for Spinless Particles Subject to a Classical Electromagnetic Field
}

\author{
by \\ Julien Sater

\begin{abstract}
Submitted to the School of Mathematics and Statistics
\end{abstract} \\ in partial fulfillment of the requirements for the degree of \\ Master of Science in Applied Mathematics
}

\begin{abstract}
The theory of Artificial Boundary Conditions described by Antoine et al. $[2,4-6]$ for the Schrödinger equation is applied to the Klein-Gordon (KG) in two-dimensions (2-D) for spinless particles subject to electromagnetic fields. We begin by providing definitions for a basic understanding of the theory of operators, differential geometry and wave front sets needed to discuss the factorization theorem thanks to Nirenberg and Hörmander $[14,16]$. The laser-free Klein-Gordon equation in 1-D is then discussed, followed by the case including electrodynamics potentials, concluding with the $\mathrm{KG}$ equation in 2-D space with electrodynamics potentials. We then consider numerical simulations of the laser-particle KG equation, which includes a brief analysis of a finite difference scheme. The conclusion integrates a discussion of the numerical results, the successful completion of the objective set forth, a declaration of the unanswered encountered questions and a suggestion of subjects for further research.
\end{abstract}


Library and Archives

Canada

Published Heritage

Branch

395 Wellington Street

Ottawa ON K1A ON4

Canada
Bibliothèque et

Archives Canada

Direction du

Patrimoine de l'édition

395 , rue Wellington

Ottawa ON K1A ON4

Canada
Your file Votre référence

ISBN: 978-0-494-94313-7

Our file Notre référence

ISBN: $978-0-494-94313-7$
NOTICE:

The author has granted a nonexclusive license allowing Library and Archives Canada to reproduce, publish, archive, preserve, conserve, communicate to the public by telecommunication or on the Internet, loan, distrbute and sell theses worldwide, for commercial or noncommercial purposes, in microform, paper, electronic and/or any other formats.

The author retains copyright ownership and moral rights in this thesis. Neither the thesis nor substantial extracts from it may be printed or otherwise reproduced without the author's permission.
AVIS:

L'auteur a accordé une licence non exclusive permettant à la Bibliothèque et Archives Canada de reproduire, publier, archiver, sauvegarder, conserver, transmettre au public par télécommunication ou par l'Internet, prêter, distribuer et vendre des thèses partout dans le monde, à des fins commerciales ou autres, sur support microforme, papier, électronique et/ou autres formats.

L'auteur conserve la propriété du droit d'auteur et des droits moraux qui protege cette thèse. $\mathrm{Ni}$ la thèse ni des extraits substantiels de celle-ci ne doivent être imprimés ou autrement reproduits sans son autorisation.
In compliance with the Canadian Privacy Act some supporting forms may have been removed from this thesis.

While these forms may be included in the document page count, their removal does not represent any loss of content from the thesis.
Conformément à la loi canadienne sur la protection de la vie privée, quelques formulaires secondaires ont été enlevés de cette thèse.

Bien que ces formulaires aient inclus dans la pagination, il n'y aura aucun contenu manquant. 


\section{Acknowledgments}

I would like to take this opportunity to express my sincere gratitude to my supervisor, Emmanuel Lorin, whose extensive knowledge of the subject and interesting variety of research topics he proposed, kept me highly captivated. His sound advice and constant support not only enhanced my motivation and focus on the task, but also considerably helped shape this work.

Likewise, I want to convey my special thanks to François Fillion-Gourdeau for raising the initial question as well as for his precious assistance in physics and programming.

I wish to thank Lucy Campbell for an excellent course in partial differential equations, and for being part of the jury presiding the defense of my thesis.

Furthermore, I also wish to thank Rémi Vaillancourt and Abhijit Sarkar for officiating the defense of my thesis and for their valuable time and input.

I am also extending my appreciation to the School of Mathematics and Statistics at Carleton University for accommodating me over the past two years.

Finally, I am truly grateful to my parents for their everlasting belief in me and their continuous endorsement. 


\section{Contents}

1 Introduction 1

2 Useful definitions $\quad 6$

2.1 Some elements of operator theory . . . . . . . . . . . . . 6

2.2 Some elements of differential geometry . . . . . . . . . . . . 9

2.3 Some notions about wave front sets . . . . . . . . . . . . . . . . 14

3 Klein-Gordon equation in 1-D $\quad 18$

3.1 The laser-free Klein-Gordon equation . . . . . . . . . . . 18

3.2 The Klein-Gordon equation with electrodynamic potentials . . . . . 26

4 Klein-Gordon equation in 2-D 32

4.1 The Klein-Gordon equation with electrodynamic potentials . . . . . 32

5 Numerical analysis $\quad 46$

5.1 Finite difference analysis . . . . . . . . . . . . . . . 47

5.2 Numerical results . . . . . . . . . . . . . . . . . 52

6 Conclusion and future considerations $\quad 56$ 


\section{Chapter 1}

\section{Introduction}

The Klein-Gordon (KG) equation is a relativistic version of the Schrödinger wave equation and arises very naturally, with additional terms, in quantum dynamics of high intensity laser-matter interactions. It governs the behaviour of a charged spinless particle, such as the Higgs boson. Although from a particle physics standpoint, the Dirac and Schrödinger equations are more interesting to study, the KG is still an important equation in the literature. If we look at it from a mathematical standpoint, then we are working with a second order wave equation with additional space and time dependent variable and thus the resolution of such equations can be relevant in a variety of fields.

In this thesis, we are interested in finding Absorbing Boundary Conditions (ABC) for a Klein-Gordon equation whose particles are subject to time and space dependent electrodynamic fields (laser pulse). These absorbing boundary conditions have for purpose to reduce the numerical reflection that can occur when imposing a finite boundary on a solution that is supported in all of $\mathbb{R}^{d}$, where $d$ is the spacial dimension. Specifically, we will build the theory in order to consider the scalar 2-D Klein-Gordon equation. We start with a case free from any laser and set in the unbounded domain 
$\mathbb{R}^{2} \times[0, \infty)$

$$
\left\{\begin{array}{l}
L \varphi:=\left(-\partial_{t}^{2}+c^{2} \partial_{x}^{2}+c^{2} \partial_{y}^{2}-m^{2} c^{4}\right) \varphi(x, y, t)=0, \quad \forall(x, y, t) \in \mathbb{R}^{2} \times[0, \infty) \\
\varphi(x, y, 0)=\varphi_{0}(x, y), \quad \forall(x, y) \in \mathbb{R}^{2}
\end{array}\right.
$$

with $\varphi_{0} \in \mathcal{L}^{2}\left(\mathbb{R}^{2}\right)$. Here $m$ and $c$ are the mass of the particle and speed of light respectively. Under the influence of electrodynamic potentials $A(x, y, t)$, a vector, and $\Phi(x, y, t)$, a scalar, the laser-particle Klein-Gordon equation reads

$$
\left\{\begin{array}{l}
L \varphi(x, y, t)=\left[\left(i \hbar \partial_{t}-e \Phi(x, y, t)\right)^{2}-c^{2}\left(\frac{\hbar}{i} \nabla-e A(x, y, t)\right)^{2}-m^{2} c^{4}\right] \varphi=0 \\
\forall(x, y, t) \in \mathbb{R}^{2} \times[0, \infty) \\
\varphi(x, y, 0)=\varphi_{0}(x, y), \quad \forall(x, y) \in \mathbb{R}^{2}
\end{array}\right.
$$

where $e$ is the charge of the particle, we note that a Coulomb potential could be added. Planck's constant $\hbar$ is introduced. The numerical approximation of (1.1) and (1.2) are usually done by truncating the unbounded domain $\mathbb{R}^{2} \times[0, \infty)$ with $\Omega \times[0, T]$, where $\Omega \subseteq \mathbb{R}^{2}$ has a smooth boundary $\Gamma=\partial \Omega$ and $0<T<\infty$. However, by doing so, the wave is then reflected at the boundary, skewing the results. The purpose of this thesis is to construct boundary conditions defined by pseudo-differential operators of the form,

$$
B\left(x, y, t, \partial_{x}, \partial_{y}, \partial_{t}\right) u(x, y, t)=\left(\partial_{n}+\Lambda\right) u=0, \quad \forall(x, y, t) \in \Gamma \times[0, T]
$$

which may be non-local in space and time, that will allow the wave to be transported through the boundary without any reflection. It will be shown that such a condition can actually be expressed by favouring the normal derivative $\left(\partial_{n}\right)$ and a first order pseudo-differential operator $(\Lambda)$ to be determined. As figure 1-1 shows, we will ap- 


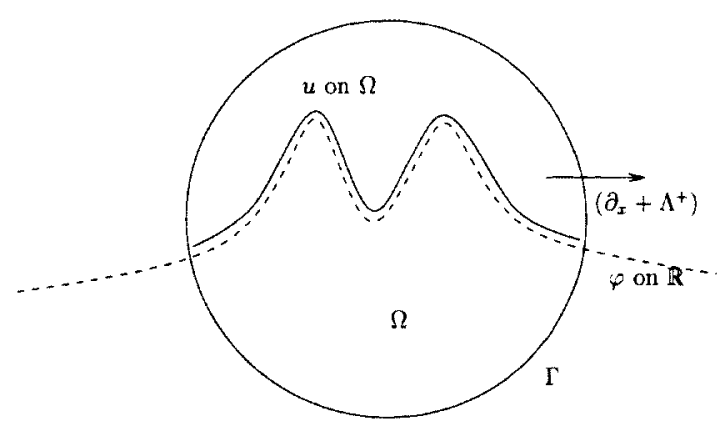

Figure 1-1: Boundary Conditions

proximate the solution $\varphi$ by $u$ such that $\varphi_{\left.\right|_{\Omega}}=u$ for the following inital boundary value problem, where $u$ is properly defined in $\Omega$

$$
\left\{\begin{array}{l}
L u(x, y, t)=\left[\left(i \hbar \partial_{t}-e \Phi(x, y, t)\right)^{2}-c^{2}\left(\frac{\hbar}{i} \nabla-e A(x, y, t)\right)^{2}-m^{2} c^{4}\right] u=0 \\
\forall(x, y, t) \in \Omega \times[0, T], \\
B\left(x, y, t, \partial_{x}, \partial_{y}, \partial_{t}\right) u=0, \quad \forall(x, y, t) \in \Gamma \times[0, T], \\
u(x, y, 0)=\varphi_{0}(x, y), \quad \forall(x, y) \in \Omega .
\end{array}\right.
$$

As stated before, the boundary conditions (1.3) are constructed to reduce the cost of the computation of the exact solution on all $\mathbb{R}^{2}$, into a viable approximation in $\Omega$. Such conditions are known as artificial boundary conditions. In the case where the artificial boundary condition is such that $u=\varphi_{\left.\right|_{\Omega}}$, we say it is a transparent boundary condition (TBC). Unfortunately, most TBCs are often non local in either space or time or both, hence the search for a well-posed condition brought about absorbing boundary conditions ( $\mathrm{ABC}$ ), when $u \simeq \varphi_{\mathrm{I}_{\Omega}}$ as described in [2]. They get their name from the sense that they are said to "absorb" some of the energy from the waves hitting the boundary. Hence, artificial boundary conditions are called ABCs when they yield a well-posed initial boundary value problem (IBVP). The well-posedness of $\mathrm{ABCs}$ allow for some locality in either space and/or time based on their construction. We will see in this thesis that the conditions constructed will be nonlocal in time, but 
will have the nice property of being local in space, partly due to the pseudo-locality of pseudo-differential operators we will introduce. Once again for more information, we refer to [2].

We note that Antoine \& Besse $[5,6]$ have recently developed efficient $\mathrm{ABC} / \mathrm{TBCs}$ for the linear and nonlinear Schrödinger equations. In our case we will show that the method can be applied on other wave equations, mainly (1.2). The origins of the transparent and absorbing boundary conditions (TBC, $\mathrm{ABC}$ ) are due to the pioneering work of Engquist \& Majda [9] who used the theory of reflection of singularities which in turn came from the work on pseudo-differential operators by Nirenberg [16] and Hörmander [11-15]. They used these operators and their associated symbols to study the reflection of waves at a boundary. This led to the possible characterization of the "incoming" and "outgoing" waves, which is possible thanks to the factorization theorem due to Nirenberg [16].

This work will be presented in the following manner: in Chapter 2, we will present with an adequate amount of detail, the implementation of the factorization theorem by Nirenberg. We start first by defining what is a pseudo-differential operator and its symbol, while listing some properties of symbolic calculus. We then look into some necessary theorems and definitions in differential geometry which allow us to link between pseudo-differential operators and the Hamiltonian equations. Chapter 2 also describes how to rewrite the equation in microlocal coordinates favouring the normal derivative and will conclude with the key concept of wave front sets, which is the factorization theorem by Nirenberg. In Chapter 3, we present the method used by Antoine \& Besse [4] adapted to the scalar 1-D Klein-Gordon equation free from any electrodynamic potentials and meant as a gentle intoduction of the steps to follow. The Riemann-Liouville integral is also defined in this Chapter as a method to deal with fractional or negative differentials that might appear during the approximation. Chapter 4 is a follow-up of Chapter 3 , that is, we now repeat the method for a laserparticle problem in 2-D, hence (1.2), which is the main result of this thesis. We will see that in the two-dimensional case, an asymptotic expansion based on high-frequencies 
will be needed and it will be shown explicitly for our two dimensional case. In Chapter 5, we will present the benchmarks of our method for a 1-D scalar Klein-Gordon equation with electrodynamic potentials. A finite difference scheme will be studied by showing the consistency and the stability conditions for the equation. We conclude with some remarks discussing the efficiency of the method. 


\section{Chapter 2}

\section{Useful definitions}

This chapter introduces some notations and definitions that will be needed throughout. It will also provide an adequate background for a basic understanding of the analysis behind the method.

Let us first start by introducing $\Omega$, an open subset of $\mathbb{R}^{n}$ with smooth boundary. We denote by $\mathbb{C}^{\infty}(\Omega)$ the set of infinitely continuously differentiable functions on $\Omega$ with values in $\mathbb{C}$, and $\mathbb{C}_{0}^{\infty}(\Omega)$ is a subspace of $\mathbb{C}^{\infty}(\Omega)$ whose elements are zero outside a compact subset of $\Omega$, hence with compact support.

For a few definitions, mainly of operators, the use of multiple indices is required. Let $\alpha=\left(\alpha_{1}, \ldots, \alpha_{n}\right)$ be an element of $\mathbb{N}^{n}$, then $|\alpha|=\alpha_{1}+\cdots+\alpha_{n}$ and we set $\alpha !=\alpha_{1} ! \ldots \alpha_{n} !$

Next, the following notation $D_{j}=-i \frac{\partial}{\partial x_{j}}$ for $j \in 1, \ldots, n$, will denote the partial derivatives with respect to $x_{j}$, while $D^{\alpha}=D_{1}^{\alpha_{1}} \ldots D_{n}^{\alpha_{n}}$ or $\partial^{\alpha}=\partial_{1}^{\alpha_{1}} \ldots \partial_{n}^{\alpha_{n}}$ are higherorder derivatives.

\subsection{Some elements of operator theory}

We now draw the following definitions from [1].

Definition 2.1.1 A differential operator on $\Omega$ is a finite linear combination of derivatives of arbitrary orders with coefficients in $\mathbb{C}^{\infty}(\Omega)$. It is said to be of order 
$m$ if it does not include derivatives of order strictly greater than $m$. In other words, a differential operator of order $m$ on $\Omega$ can be written as

$$
P=\sum_{|\alpha| \leq m} a_{\alpha}(x) D^{\alpha}
$$

where the $a_{\alpha} \in \mathbb{C}^{\infty}(\Omega)$ are the coefficients of $P$.

Definition 2.1.2 The symbol $p$ of a differential operator $P$ (2.1) is defined as the polynomial function in $\xi$ on $\Omega \times \mathbb{R}^{n}$ by

$$
p(x, \xi)=\sum_{|\alpha| \leq m} a_{\alpha}(x) \xi^{\alpha}
$$

while its principal symbol of order $m$ or principal symbol is the homogenous function in $\xi$ of order $m$

$$
p_{m}(x, \xi)=\sum_{|\alpha|=m} a_{\alpha}(x) \xi^{\alpha}
$$

Next, we discuss how differential operators can be used in conjunction with the Fourier transform to become a powerful tool for use in the study of partial differential equation. First we must introduce the space of rapidly decreasing functions in which the symbols associated to operators exist, and we refer to [12] for more details.

Definition 2.1.3 The space $\mathcal{S}$ of $\mathbb{C}^{\infty}$ functions which decrease rapidly on $\mathbb{R}^{n}$ is the space of $\mathbb{C}^{\infty}$ functions $u$ on $\mathbb{R}^{n}$, satisfying

$$
\forall \alpha \in \mathbb{N}^{n}, \forall \beta \in \mathbb{N}^{n}, \sup _{x \in \mathbb{R}^{n}}\left|x^{\alpha} \partial^{\beta} u(x)\right|<+\infty
$$

The space $\mathcal{S}$ is then provided with the semi-norms defined in this way, as $\alpha$ and $\beta$ run over $\mathbb{N}^{n}$. If $x=\left(x_{1}, \ldots, x_{n}\right)$ is a vector of $\mathbb{R}^{n}$, we set

$$
|x|^{2}=\sum_{j=1}^{n} x_{j}^{2}
$$


Definition 2.1.4 Let $m \in \mathbb{R}$. Let $S^{m}=S^{m}\left(\mathbb{R}^{n} \times \mathbb{R}^{n}\right)$ denote the set of $a \in \mathbb{C}^{\infty}\left(\mathbb{R}^{n} \times\right.$ $\left.\mathbb{R}^{n}\right)$ such that

$$
\forall \alpha, \forall \beta,\left|\partial_{x}^{\alpha} \partial_{\xi}^{\beta} a(x, \xi)\right| \leq C_{\alpha, \beta}(1+|\xi|)^{m-|\beta|}
$$

we also denote $S^{-\infty}=\cap_{m} S^{m}$. An element $a \in S^{m}$ is called a symbol of order $m$.

We now discuss more formally operators called pseudo-differential operators. Once again, we refer to [1] for details.

Proposition 2.1.1 If $p_{m} \in S^{m}$ and $u \in \mathcal{S}$, the formula

$$
O p\left(p_{m}\right) u(x)=\frac{1}{(2 \pi)^{n / 2}} \int_{\mathbb{R}^{n}} e^{i x \xi} p_{m}(x, \xi) \hat{u}(\xi) \mathrm{d} \xi
$$

defines a function of $\mathcal{S}$.

Definition 2.1.5 For $p \in S^{m}$, the operator $O p\left(p_{m}\right)$ is the pseudo-differential operator with symbol $p_{m}$. A pseudo-differential operator is said to be of order $m$ if its symbol belongs to $S^{m}$.

We now list a few useful properties and notations of pseudo-differential operators and symbols. We say that $O p(a)$ is an operator whose symbol is $a$ while $\sigma(A)$ is a symbol whose operator is $A$. Moreover, the multiplication of symbols is given by

Theorem 2.1.1 If $a_{1} \in S^{m_{1}}, a_{2} \in S^{m_{2}}$, we have $O p\left(a_{1}\right) O p\left(a_{2}\right)=O p(b)$, where $b=a_{1} \# a_{2} \in S^{m_{1}+m_{2}}$ is given by

$$
b(x)=(2 \pi)^{-n / 2} \int e^{-i x \xi} a_{1}(x) a_{2}(x) \mathrm{d} \xi
$$

and $b \sim \sum_{\alpha=0}^{\infty} \frac{(-i)^{\alpha}}{\alpha !} \partial_{\xi}^{\alpha} a_{1} \partial_{x}^{\alpha} a_{2}$. We also note that $\sigma(A B)=\sigma(A) \# \sigma(B)$

We refer to Nirenberg [16] for details, but this can be determined with the help of Leibniz' formula. 
For the purpose of this thesis, we shall restrict ourselves to a smaller class then definition 2.1.4, which in turn is smaller than the oscillatory integrals due to Hörmander [12]. This will allow for an asymptotic expansion as shown in [16]. Consider $\mathbb{C}^{\infty}$ functions $p(x, \xi)$, satisfying: for each $p$ there is an integer $m$, the order of the operator, and a sequence $p_{m-j}(x, \xi)$, for $j \in \mathbb{N}$ with $\xi \neq 0$ such that

$$
D_{x}^{\beta}\left(p-\sum_{0}^{n} p_{m-j}\right)=0\left(|\xi|^{m-n-1}\right), \quad|\xi| \rightarrow \infty
$$

$\forall n \in \mathbb{N}$ and for any $\beta$ multi index. We write

$$
p(x, \xi) \sim \sum_{0}^{\infty} p_{m-j}(x, \xi)
$$

We assure here that $p_{m-j}$ is a homogeneous polynomial in $\xi$, that is to say a polynomial whose variables with nonzero coefficients all have the same total degree. We introduce this more formal definition

Definition 2.1.6 A symbol $p \in S^{m}$ is said to be classical if $p \sim \sum_{j \geq 0} p_{m-j}$, where the functions $p_{m-j}$ are homogeneous of degree $m-j$ for $|\xi| \geq 1$, that is $p_{m-j}(x, \lambda \xi)=$ $\lambda^{m-j} p_{m-j}(x, \xi)$ for $|\xi| \geq 1, \lambda \geq 1$.

The need for this class of symbol will be relevant later on, when we construct our absorbing boundary conditions.

\subsection{Some elements of differential geometry}

We refer to [1] for our discussion on differentiable manifolds. The use of differential manifolds is essential to describe how the characteristics of our equations behave, so we make some brief recalls. We start by showing that our boundary conditions can be written with the help of pseudo-differential operators microlocally favouring the 


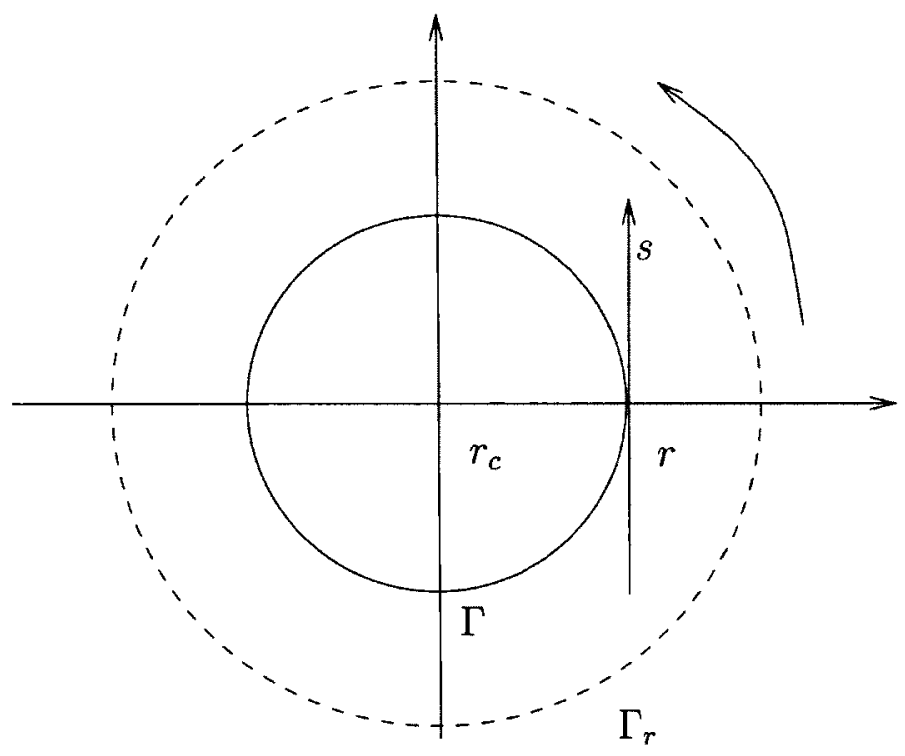

Figure 2-1: Microlocal Coordinates at the Boundary

normal direction, i.e.:

$$
B u=\left(\partial_{n}+\widetilde{\Lambda}^{+}\right) u=0, \text { on } \Gamma \times[0, T]
$$

where $\widetilde{\Lambda}^{+}=\widetilde{\Lambda}^{+}\left(t, x, \partial_{t}, \partial_{x}\right) \in O P S^{m}$. Here $\Gamma$ is the boundary of $\Omega$, while $\Gamma_{r}$ is a boundary that incloses it where $0<r<\epsilon$ for $\epsilon>0$ small. Variables $(r, s)$ designate the new local coordinates, $r$ is in the radial direction, and $s=r_{\mathrm{c}} \theta$ is the curvilinear abscissa on $\Gamma$, see figure 2-1. It is worth noting here that if $r=0$ then $\Gamma_{r}=\Gamma_{0}=\Gamma$. Hence the Laplacian under these coordinates is $\Delta_{r, s}=\partial_{r}^{2}+\kappa_{r} \partial_{r}+h^{-1} \partial_{s}\left(h^{-1} \partial_{s}\right)$, where $\kappa_{r}=h^{-1} \kappa, \kappa$ being the curvature and $h(r)=1+r \kappa$, we refer to [3] for more details. In our case, since we choose $\Omega$ to be a simple circle with constant radius $r_{c}$, thus the curvature is also constant, and we actually obtain

$$
\Delta_{r, \theta}=\partial_{r}^{2}+\frac{1}{R} \partial_{r}+\frac{1}{R^{2}} \partial_{\theta}^{2}
$$

since $\partial_{s}^{2}=\frac{1}{r_{c}^{2}} \partial_{\theta}^{2}, R=r_{c}+r$ and $\theta \in[0,2 \pi]$.

We are now in need to introduce some concepts of tangent and cotangent bundles. 
Throughout, we denote by $X$ a $\mathbb{C}^{\infty}$-manifold of dimension $n$, and we refer to [1] for more details.

Definition 2.2.1 A tangent bundle of a differentiable manifold $X$ is the disjoint union of the tangent spaces of $X$, that is the collection of all tangent vectors. Formally:

$$
T(X)=\bigcup_{x \in X}\{x\} \times T_{x}(X)
$$

where $T_{x}(X)$ denotes the tangent space to $X$ at the point $x$ and is of dimension $2 n$.

On this tangent space we can define a 1-form, or covector, denoted by $\omega=\sum_{i}^{n} \xi_{i} \mathrm{~d} x_{i}$ where $\left(\mathrm{d} x_{i}\right)_{i}$ make up the basis of the space $T_{x}^{*}(X)$ which is simply dual space of $T_{x}(X)$. For more detail, we refer to [7] or any other good book in differential geometry. We now introduce the notion of the cotangent bundle

Definition 2.2.2 The cotangent bundle of a manifold $X$

$$
T^{*}(X)=\bigcup_{x \in X}\{x\} \times T_{x}^{*}(X)
$$

is the dual bundle to the tangent bundle.

Now we need to extract the notion of 2 -form in order to make the link with the Hamiltonian vector field. Briefly put, if we repeat this process with a element $Z$ of $T_{(x, \omega)}\left(T^{*}(X)\right)$ with a covector $\alpha_{(x, \omega)}=\sum_{j} \alpha_{j} \mathrm{~d} x_{j}$ that acts on the tangent bundle of the cotangent bundle of $X$, that is $T_{(x, \omega)}\left(T^{*}(X)\right)$. We note here that $\operatorname{dim}\left(T^{*}(X)\right)=2 n$, hence we can write the vector $Z=\sum_{i}^{n} z_{1, i} \frac{\partial}{\partial x_{i}}+\sum_{i}^{n} z_{2, i} \frac{\partial}{\partial \xi_{i}}$ where $\partial_{x_{i}}, \partial_{\xi_{i}}$ is the basis of $T_{(x, \omega)}\left(T^{*}(X)\right)$. Now we introduce a 2-form $\sigma_{(x, \omega)}(Z, Y)=\mathrm{d} \omega=a_{z} b_{y}-b_{z} a_{y}$, where $a_{z}$ is the first coefficient of $Z$ and so on. This 2 -form provides a bijection between the 1 -forms and the vector fields on $T^{*}(X)$, such that $\sigma_{(x, \omega)}(Z, Y)=\omega(Y)$. Now in the special case where $\omega=\mathrm{d} f$ for a given function $f$ on $T^{*}(X)$, then $Z$ is called the Hamiltonian vector field and $\omega(Y)=a_{y} \frac{\partial f}{\partial x}+b_{y} \frac{\partial f}{\partial \xi}$. It is now possible to match 
$\sigma(Z, Y)$, therefore

$$
\sigma(Z, Y)=a_{z} b_{y}-b_{z} a_{y}=a_{y} \frac{\partial f}{\partial x}+b_{y} \frac{\partial f}{\partial \xi}
$$

and we obtain $a_{z}=\frac{\partial f}{\partial \xi}$ and $b_{z}=-\frac{\partial f}{\partial x}$ hence

$$
Z=\frac{\partial f}{\partial \xi} \frac{\partial}{\partial x}-\frac{\partial f}{\partial x} \frac{\partial}{\partial \xi}
$$

We can now defined a bicharacteristic strip,

Definition 2.2.3 A solution of the Hamilton equations

$$
r^{\prime}(s)=\frac{\partial p_{m}}{\partial \rho}, \rho^{\prime}(s)=-\frac{\partial p_{m}}{\partial r}
$$

for which $p_{m}(r, \rho)=0$, but $\frac{\partial p_{m}}{\partial \rho} \neq 0$ is called a null-bicharacteristic strip; the curve described by $r$ is called a bicharacteristic curve.

To this definition taken from [11], we make a remark.

1. It is sufficient to assume that $p_{m}(r, \rho)=0$ at one point of the strip for any $p_{m}(r, \rho)=0$ along the entire strip.

We will see in the next section that the set of all bicharacteristic curves emanating from $p_{m}(r, \rho)=0$ is called the wavefront set, and is contained in the hyperbolic region of the operator. We once again refer to [1] for more details.

The factorization theorem, to be shown in section 2.3 , only holds in a relevant subset of frequencies. Let us explain:

let the Klein-Gordon operator $P=-\partial_{t}^{2}+c^{2} \partial_{r}^{2}+\frac{1}{R} \partial_{r}+\frac{1}{R^{2}} \partial_{\theta}^{2}-m^{2} c^{4}$ be in the domain described by figure 2-1. We then search for when the principal symbol 


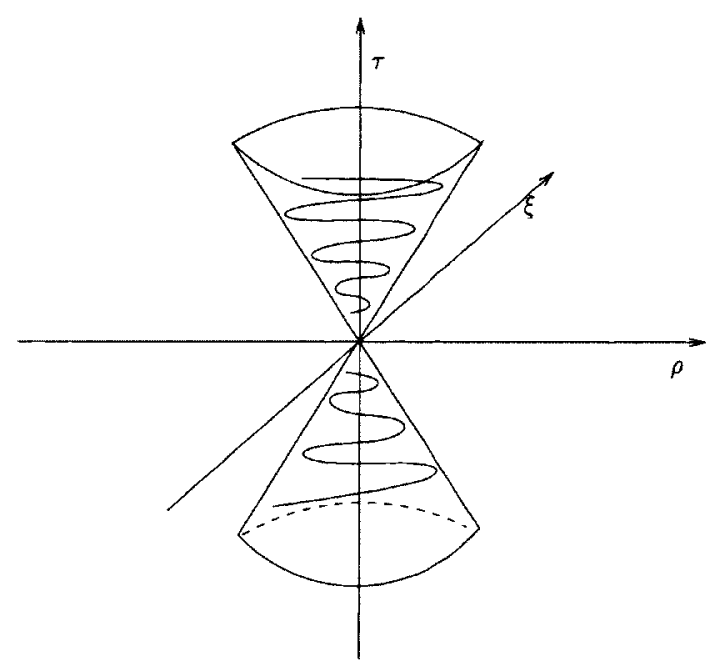

Figure 2-2: Hyperbolic Region 3-D

$p_{2}(t, r, \theta, \tau, \rho, \xi)=0$ at the boundary, $p_{2}$ since $m=2$. Hence the principal symbol at $r=0$ is

$$
p_{2}\left(t_{0}, 0, \theta_{0}, \tau_{0}, \rho_{0}, \xi_{0}\right)=\tau_{0}^{2}-\rho_{0}^{2}-\xi_{0}^{2}=0
$$

here $(\tau, \rho, \xi)$ represent the covariables of $(t, r, \theta)$ respectively with $(\tau, \rho, \xi, t, r, \theta) \in$ $T^{*}(\Omega \times[0, T])$. This second-order equation with respect to $r$ admits two roots $\rho_{0}=$ $\pm \sqrt{\tau_{0}^{2}-\xi_{0}^{2}}$, and helps to define the hyperbolic region when $\tau_{0}^{2}-\xi_{0}^{2}>0$, hence $\rho_{0}$ has real roots. We can now more formally characterize the set of frequencies for which the factorization holds: the hyperbolic region is $\mathcal{H}=\left\{\left(t_{0}, \theta_{0}, \tau_{0}, \xi_{0}\right) \in T^{*}(X) \mid \tau_{0}^{2}-\xi_{0}^{2}>0\right\}$. Similarly, the elliptic region is defined as $\mathcal{E}=\left\{\left(t_{0}, \theta_{0}, \tau_{0}, \xi_{0}\right) \in T^{*}(X) \mid \tau_{0}^{2}-\xi_{0}^{2}<0\right\}$, and the solutions to $p_{2} \hat{u}=0$ are evanescent. The shaded region in figure (2-2) demonstrates the hyperbolic region of interest. We refer to [4] for more details. However, before we can truly sum up the theory, we need more details on how these characteristics behave in the space of distributions. 


\subsection{Some notions about wave front sets}

We first introduce the concept of support and singular support, as shown in [1]. The wave front set is an extension of the singular support of a function. In the space of distributions on $\Omega$, denoted by $\mathcal{D}^{\prime}(\Omega)$, we define the support of $u \in \mathcal{D}^{\prime}(\Omega)$ to be the complement in $\Omega$ of the points in the neighbourhood of which $u$ is zero. The support of $u$ is denoted by supp $u$.

$$
u=0 \Leftrightarrow \operatorname{supp} u=\emptyset
$$

The singular support of a distribution $u \in \mathcal{D}^{\prime}(\Omega)$ is defined to be the complement in $\Omega$ of the points in the neighbourhood of which $u$ is of class $\mathbb{C}^{\infty}$. The singular support of $u$ is denoted by sing supp $u$.

$$
u \in \mathbb{C}^{\infty} \Leftrightarrow \operatorname{sing} \operatorname{supp} u=\emptyset
$$

An intuitive interpretation of the sing supp $u$ is that of the set of points at which a distribution fails to be a smooth function. Finally, the space of distributions with compact support in $\Omega$ is denoted by $\mathcal{E}^{\prime}(\Omega)$, which is compliant with the space in which our operators exist. Here we restate that condition more explicitly for the Fourier transform, in order to properly present the theory as described by [1]:

The Fourier transform $\hat{u}$ of a function $u \in \mathbb{C}_{0}^{\infty}\left(\mathbb{R}^{n}\right)$ is rapidly decreasing in the Fourier coefficient $\xi$, that is

$$
\forall k \in \mathbb{N},|\hat{u}(\xi)| \leq C_{k}(1+|\xi|)^{-k}
$$

for any positive constant $C_{k}$.

Conversely, if (2.21) is satisfied by the Fourier transform of a distribution $u \in$ $\mathcal{E}^{\prime}\left(\mathbb{R}^{n}\right)$, then $u \in \mathbb{C}_{0}^{\infty}\left(\mathbb{R}^{n}\right)$, because of the Fourier inversion formula.

Definition 2.3.1 For $u \in \mathcal{E}^{\prime}\left(\mathbb{R}^{n}\right)$, let $\Sigma(u)$ be the complement of the set of directions 
$\xi \in \mathbb{R}^{n} \backslash\{0\}$ in the conical section of which $\hat{u}$ satisfies (2.21). Conical is defined as $\xi \in \Omega, \lambda>0 \Rightarrow \lambda \xi \in \Omega$, which is what figure (2-2) shows in 3-D.

We can think of things this way, just as the sing supp $u$ can be considered as the set of 'bad points' of $u$, then $\Sigma(u)$ is the set of 'bad directions' or 'bad frequencies' of $u$. Next we need the following lemma from [1] and we refer to (p.89) for the proof.

Lemma 2.3.1 If $\varphi \in \mathbb{C}_{0}^{\infty}\left(\mathbb{R}^{n}\right)$ and $u \in \mathcal{E}^{\prime}\left(\mathbb{R}^{n}\right), \quad \Sigma(\varphi u) \subset \Sigma(u)$

We can finally introduce the wave front set of $u$ :

Definition 2.3.2 Let $X$ be an open subset of $\mathbb{R}^{n}$ and $u \in \mathcal{D}^{\prime}(X)$. The closed conical subset of $X \times\left(\mathbb{R}^{n} \backslash\{0\}\right)$ defined by

$$
W F(u)=\left\{(x, \xi) \in X \times\left(\mathbb{R}^{n} \backslash\{0\}\right), \xi \in \Sigma_{x}(u)\right\}
$$

is the wave front set $u$.

The following proposition shows that $W F(u)$ is the of 'bad directions' of $u$ over sing supp $u$.

Proposition 2.3.1 The projection of $W F(u)$ onto $X$ is equal to sing supp $u$.

We now formally state that pseudo-differential operators have the following property; Any pseudo-differential operator $A$ that satisfies (2.7)

$$
\text { sing supp Au } \subset \text { sing supp } u \text {. }
$$

is said to be pseudo-local. We refer to [16] for the proof.

Now thanks to proposition 2.3 .1 and definition 2.3 .2 we obtain the following corollary.

Corollary 2.3.1 Let $A$ be a pseudo-differential operator. Then for all $u \in \mathcal{E}^{\prime}\left(\mathbb{R}^{n}\right)$, $W F(A u) \subset W F(u)$. 
Another way to view this last corollary is that the actions of a pseudo-differential operator keep the wave front set stable. This essentially provides us with twice as much information about our functions as before, we get some information from the regular space, while the other information comes from the dual space.

Let us put things into perspective:

- We showed the link between the Fourier transform and pseudo-differential operators.

- Those pseudo-differential operators may have principal symbols (2.3), which have an asymptotic expansion (2.10) when $p_{m-j}$ is homogeneous in $\xi$.

- We then defined the cotangent bundle, which was used to link the symbols to the Hamilton equations.

- The solutions to those equations describe the bicharacteristics curves (definition 2.2.3) where solution travels along the strip.

- The wave front set (definition 2.3.2) is shown to be in the hyperbolic region in which the solutions propagate.

- Finally, we showed that wave front set remains undisturbed by the actions of a pseudo-differential operator (corollary 2.3.1) thanks, in part, to their pseudolocal property.

Now all that it left to show is one of the more fundamental results, and is due to Hörmander $[12,14]$. The theorem is stated without proof from [1] (p.102)

Theorem 2.3.1 Let $P$ be a pseudo-differential operator of order $m$, which is properly supported on an open set $X \subset \mathbb{R}^{n}$ and has real principal symbol $p_{m}$. If $u$ is in $\mathcal{D}^{\prime}(X)$ and satisfies $P u \in \mathbb{C}^{\infty}(X)$, then $W F(u)$ is a union of the bicharacteristic curves emanating from points $(x, \xi)$ where $p_{m}(x, \xi)=0$.

This theorem allows us to seperate the rays of propagation of singularies starting from a point $x_{0} \in \operatorname{sing} \operatorname{supp} u$. 
We can now finally state the factorization theorem which will be used in the coming chapters.

Theorem 2.3.2 When $x \in \mathbb{R}$, a differential operator of order $m$,

$$
P\left(t, x, D_{t}, D_{x}\right)=\sum_{k+l \leq m} a_{k, l} D_{x}^{k} D_{t}^{l}
$$

can be factorized $\forall j<m$, starting at $j=1$, in the following form

$$
P\left(t, x, D_{t}, D_{x}\right)=\left(D_{x}-\lambda_{1}(t, x) D_{t}\right) \ldots\left(D_{x}-\lambda_{m}(t, x) D_{t}\right)+\text { order } m-j
$$

where the $\lambda_{j} \xi$ are the roots in $\tau$ (assumed distinct) of the polynomial

$$
p_{m}=\sum_{k+l=m} a_{k, l} \xi^{k} \tau^{l}=0
$$

It is worth noting that there are as many 'factors' as the order of the symbol, i.e., when $m=2$, we have our 'incoming' and 'outgoing' directions for our waves. We remark also that the theory only permits us to describe the behaviour of the solutions on the bicharacteristics for the principal symbol alone. The remaining degrees become a smoothing operator.

The next chapter will demonstrate how we use this to help construct the boundary condition operator and absorb the reflections, and where possible, make it exact. 


\section{Chapter 3}

\section{Klein-Gordon equation in 1-D}

In this section, we put into use the theory discussed in the previous chapter for two examples of the Klein-Gordon equation in 1-D. The first will be a basic laser-free Klein-Gordon equation, while the second will be an example that includes electrodynamic potentials.

\subsection{The laser-free Klein-Gordon equation}

We start by considering the case free from any electrodynamic potentials; it reads as such

$$
\left\{\begin{array}{l}
{\left[-\partial_{t}^{2}+c^{2} \partial_{x}^{2}-m^{2} c^{4}\right] \varphi(x, t)=0, \quad(x, t) \in \mathbb{R} \times[0, T]} \\
\varphi(x, 0)=\varphi_{0}(x), \quad x \in \mathbb{R}
\end{array}\right.
$$

We introduce a bounded domain $\Omega \subsetneq \mathbb{R}$ which is simply an interval and $\Gamma=\partial \Omega$ is the boundary. In local coordinates, we get

$$
\left\{\begin{array}{l}
{\left[-\partial_{t}^{2}+c^{2} \partial_{x}^{2}-m^{2} c^{4}\right] u(x, t)=0, \quad(x, t) \in \Omega \times[0, T],} \\
B u=\left(\partial_{x}+\Lambda\right) u=0, \quad(x, t) \in \Gamma \times[0, T], \\
u(x, 0)=\varphi_{0}(x), \quad x \in \Omega .
\end{array}\right.
$$


We note here that varphi $i_{0}(x)$ is assumed to be properly supported in $\Omega$. The first thing to do is to determine the bicharacteristic strips of our equation in order to isolate the reflected waves. So with our operator $P\left(t, x, D_{t}, D_{x}\right)$ and the following dual variables $(\tau, \xi)$ for $(x, t)$ respectively, we want to obtain the principal symbol of our Klein-Gordon equation (3.2). If we take the Fourier transform, then

$$
p_{m}(t, x, \tau, \xi) \hat{\varphi}(\tau, \xi)=\left[\tau^{2}-c^{2} \xi^{2}-m^{2} c^{4}\right] \hat{\varphi}(\tau, \xi)
$$

We see here that the order of the principal symbol is $m=2$ and we expect to find two roots of $p_{m}=0$ at the boundary, meaning when

$$
\begin{aligned}
& p_{2}\left(t_{0}, 0, \tau_{0}, \xi_{0}\right)=\tau_{0}^{2}-c^{2} \xi_{0}^{2}=0 \\
& \xi^{ \pm}= \pm \frac{1}{c} \sqrt{\tau^{2}}
\end{aligned}
$$

We obtain two roots $\xi^{ \pm}$as desired, and they help characterize the 'incoming' and 'outgoing' wave directions. Now for our solutions to be non-trivial, we recall that we want real roots.

The next step is to choose the correct direction based on the Hamilton equations just as was done in Chapter 2. As we want the forward moving equation to proceed through the boundary without any reflections, we require:

$$
\dot{x}(s)=\frac{\partial}{\partial \xi} p_{2}(s)=-2 c^{2} \xi_{0}>0 .
$$

So with $\tau$ real and $\xi_{0}^{ \pm}= \pm \tau / c$ the choice becomes obvious. Let $\xi_{0}^{+}=-\tau / c$ be the solution carried across the forward bicharacteristic curve. Now comes the use of the factorization theorem (2.3.2) which initially originated from Nirenberg [16] and as was used in by Antoine et. al. [4-6] for the linear and nonlinear Schrödinger equation. We will explicitly determine the symbols of the factorization by matching them with the symbols of the Klein-Gordon equation. From there, the use of the inverse Fourier tranform, and if needed, the Riemann-Liouville integral for fractional or negative 
derivates will be used to revert back to the operators. So by definition (2.3.2) we can say that the principal symbol of (3.3) can be factorized as such

$$
L\left(t, x, \partial_{t}, \partial_{x}\right)=\left(c \partial_{x}-\Lambda^{-}\left(t, x, \partial_{t}\right)\right)\left(c \partial_{x}+\Lambda^{+}\left(t, x, \partial_{t}\right)\right)+R\left(t, x, \partial_{t}\right)
$$

here $R\left(t, x, \partial_{t}\right)$ is a smoothing operator and its symbol $\sigma(R) \in S^{-\infty}$. We will denote by $\lambda^{+}=\sigma\left(\Lambda^{+}\right)$as the symbol of the 'outgoing wave' operator and $\lambda^{-}=\sigma\left(\Lambda^{-}\right)$is the 'incoming wave' operator. Now we have a condition that helps us determine which one of these is the outgoing wave, namely $\xi_{0}^{+}=-\tau_{0} / c$, so we take a similar decomposition of $p_{2}(\tau, \xi)=-c^{2}\left(\xi-\xi_{0}^{-}\right)\left(\xi-\xi_{0}^{+}\right)=0$ and match it to the symbols of (3.7) $p_{2}=\sigma(L)$, therefore

$$
\begin{aligned}
-c^{2}\left(\xi-\xi_{0}^{-}\right)\left(\xi-\xi_{0}^{+}\right) & =\left(i c \xi-\lambda^{-}(\tau, \xi)\right)\left(i c \xi+\lambda^{+}(\tau, \xi)\right)=0 \\
\left(c \xi-c \xi_{0}^{-}\right)\left(c \xi-c \xi_{0}^{+}\right) & =\left(c \xi+i \lambda^{-}\right)\left(c \xi-i \lambda^{+}\right)=0 \\
\left(c \xi-c \xi_{0}^{+}\right) & =\left(c \xi-i \lambda^{+}\right) \\
c \xi_{0}^{+} & =i \lambda^{+}
\end{aligned}
$$

which means we are looking for when

$$
-\tau_{0}=i \lambda^{+} \Rightarrow i \tau_{0}=\lambda^{+}
$$

in order to determine the outgoing wave. Now let us develop (3.7):

$$
\begin{aligned}
L & =\left(c \partial_{x}-\Lambda^{-}\right)\left(c \partial_{x}+\Lambda^{+}\right)+R \\
& =c^{2} \partial_{x}^{2}+c \partial_{x} \Lambda^{+}-c \Lambda^{-} \partial_{x}-\Lambda^{-} \Lambda^{+}+R \\
& =c^{2} \partial_{x}^{2}+c\left(\Lambda^{+}-\Lambda^{-}\right) \partial_{x}+c O p\left(\partial_{x} \lambda^{+}\right)-\Lambda^{-} \Lambda^{+}+R
\end{aligned}
$$


where $O p\left(\partial_{x} \lambda^{+}\right)$comes from taking $\partial_{x} \Lambda^{+}$and applying the product rule as such:

$$
\begin{aligned}
& \partial_{x} \int e^{-i t \tau} \lambda^{+}(t, x, \tau, \xi) \hat{\varphi}(t, x, \tau, \xi) \mathrm{d} \tau= \\
& \int e^{-i t \tau}\left(\partial_{x} \lambda^{+}(t, x, \tau, \xi) \hat{\varphi}(t, x, \tau, \xi)+\lambda^{+} \partial_{x}(t, x, \tau, \xi) \hat{\varphi}(t, x, \tau, \xi)\right) \mathrm{d} \tau
\end{aligned}
$$

The equation given in (3.15) is now in the form of equation (3.2), hence it is natural to equate them up to $R$. In fact, this is what will allow us to determine the symbols explicitly, which in turn will lead to the operator of the boundary condition desired,

$$
L=-\partial_{t}^{2}+c^{2} \partial_{x}^{2}-m^{2} c^{4}=c^{2} \partial_{x}^{2}+c\left(\Lambda^{+}-\Lambda^{-}\right) \partial_{x}+c O p\left(\partial_{x} \lambda^{+}\right)-\Lambda^{-} \Lambda^{+}+R
$$

We now see the convenience of having included the speed of light $c$ in our factorization as the second order partial derivative in $x$ cancels out, and we are left with

$$
-\partial_{t}^{2}-m^{2} c^{4}=c\left(\Lambda^{+}-\Lambda^{-}\right) \partial_{x}+c O p\left(\partial_{x} \lambda^{+}\right)-\Lambda^{-} \Lambda^{+}
$$

The next step of the method is to match the orders of the equation with each other as such

$$
\left\{\begin{array}{l}
c\left(\Lambda^{+}-\Lambda^{-}\right) \partial_{x}=0, \\
c O p\left(\partial_{x} \lambda^{+}\right)-\Lambda^{-} \Lambda^{+}=-\partial_{t}^{2}-m^{2} c^{4},
\end{array}\right.
$$

and symbolically we obtain

$$
\left\{\begin{array}{l}
c\left(\lambda^{+}-\lambda^{-}\right)=0, \\
c \partial_{x} \lambda^{+}-\lambda^{-} \# \lambda^{+}=\tau^{2}-m^{2} c^{4},
\end{array}\right.
$$

where we recall theorem 2.1.1. In this case, laser-free, we note that $\sigma\left(\Lambda^{-} \Lambda^{+}\right)=\lambda^{-} \lambda^{+}$, and we get

$$
\begin{cases}\lambda^{+} & =\lambda^{-}, \\ -\lambda^{-} \lambda^{+} & =\tau^{2}-m^{2} c^{4},\end{cases}
$$




$$
\begin{cases}\lambda^{+} & =\lambda^{-} \\ -\left(\lambda^{+}\right)^{2} & =\tau^{2}-m^{2} c^{4}\end{cases}
$$

and we can see that

$$
\begin{aligned}
\left(\lambda^{+}\right)^{2} & =-\tau^{2}+m^{2} c^{4}, \\
\lambda^{+} & = \pm \sqrt{-\tau^{2}+m^{2} c^{4}} .
\end{aligned}
$$

Unfortunately, the square root proves to be problematic here as it poses an obstacle to the proper calculations of the symbols. To circumvent this nuisance, we recall that we are searching for classical symbols based on definition 2.1.6. In practice, one can use $m-j / 2$ if fractional derivatives are anticipated, as done in [4] with the Schrödinger equation. For our case here, we start with $m=2$, hence

$$
\lambda \sim \sum_{j=1}^{\infty} \lambda_{2-j}
$$

At this time, let us work it out again with our asymptotic expansion

$$
\begin{cases}c \sum_{j=1}^{n}\left(\lambda_{2-j}^{+}-\lambda_{2-j}^{-}\right) & =0 \\ c \partial_{x} \sum_{j=1}^{n} \lambda_{2-j}^{+}-\sum_{\alpha} \frac{(-i)^{\alpha}}{\alpha !} \partial_{\tau, \xi}^{\alpha} \lambda_{2-j}^{-} \partial_{t, x}^{\alpha} \lambda_{2-j}^{+} & =\tau^{2}-m^{2} c^{4}\end{cases}
$$

It is now possible to work out the terms of the multiplication, keeping in mind that the orders of symbols add with each other. Hence we can finally decompose the equation into equivalence classes, while remembering that we always consider the highest order possible. Thus, we obtain the following equations that allows us to explicitly determine the symbols of our operator:

$$
\begin{cases}c \lambda_{1}^{+}-c \lambda_{1}^{-} & =0 \\ -\lambda_{1}^{-} \lambda_{1}^{+} & =\tau^{2}\end{cases}
$$

which becomes the leading order. This was done by considering that the symbols for the first equation are of order ' +1 ', while the second is of order ' +2 ' since we add up 
the orders by symbolic multiplication. The notation order ' +1 ' is being used in this fashion because when $m-j$ becomes negative, it will be easier to say order ' -1 '. We can also see that the strength of our approximations are based on how far we allow for the expansion. After a few manipulations, we get:

$$
\left\{\begin{array}{l}
\lambda_{1}^{+}=\lambda_{1}^{-} \\
\lambda_{1}^{+}= \pm i \tau
\end{array}\right.
$$

As was done previously, we statisfy (3.6), via (3.12), hence $\lambda_{1}^{+}=i \tau$ gives us the leading order of the symbol $\lambda^{+}$. Next, we do it at order ' 0 ',

$$
\begin{cases}c \lambda_{0}^{+}-c \lambda_{0}^{-} & =0 \\ c \partial_{x} \lambda_{1}^{+}-\lambda_{1}^{-} \lambda_{0}^{+}-\lambda_{0}^{-} \lambda_{1}^{+}+i \partial_{\tau, \xi} \lambda_{1}^{-} \partial_{t, x} \lambda_{1}^{+} & =0\end{cases}
$$

and again with some manipulations,

$$
\begin{cases}\lambda_{0}^{+} & =\lambda_{0}^{-} \\ -2 \lambda_{1}^{+} \lambda_{0}^{+} & =0\end{cases}
$$

hence

$$
\lambda_{0}^{+}=0
$$

Things will not always be this convenient, but we take one more important step before defining a recursive formula. So we now take the order '-1' terms

$$
\begin{cases}c \lambda_{-1}^{+}-c \lambda_{-1}^{-} & =0 \\ c \partial_{x} \lambda_{0}^{+}-\lambda_{1}^{-} \lambda_{-1}^{+}-\lambda_{0}^{-} \lambda_{0}^{+}-\lambda_{-1}^{-} \lambda_{1}^{+} & \\ +i \partial_{\tau, \xi} \lambda_{1}^{-} \partial_{t, x} \lambda_{0}^{+}+i \partial_{\tau, \xi} \lambda_{0}^{-} \partial_{t, x} \lambda_{1}^{+}+\frac{1}{2} \partial_{\tau, \xi}^{2} \lambda_{1}^{-} \partial_{t, x}^{2} \lambda_{1}^{+} & =-m^{2} c^{4}\end{cases}
$$

and we can see that in the case of no external potential, all the partial derivative 
terms are 0 , hence we obtain

$$
\begin{gathered}
\begin{cases}\lambda_{-1}^{+} & =\lambda_{-1}^{-}, \\
-2 \lambda_{1}^{+} \lambda_{-1}^{+} & =-m^{2} c^{4},\end{cases} \\
\left\{\begin{array}{l}
\lambda_{-1}^{+}=\lambda_{-1}^{-}, \\
\lambda_{-1}^{+}=\frac{m^{2} c^{4}}{2 \lambda_{1}^{+}}
\end{array}\right.
\end{gathered}
$$

and therefore we obtain $\lambda_{-1}^{+}=-i m^{2} c^{4} / 2 \tau$. This is as expected, since the '-1' power of $\tau$ is the same as the order of our $\lambda^{ \pm}$. All further order can be obtained recursively as such

$$
\begin{cases}c \lambda_{-j}^{+}-c \lambda_{-j}^{-} & =0 \\ c \partial_{x} \lambda_{-j+1}^{+}-\sum_{l=0}^{j+1} \lambda_{1-l}^{-} \lambda_{-j+l}^{+} & =0\end{cases}
$$

where $j \geq 2$, which leads to

$$
\lambda_{-j}^{+}=\frac{1}{2 \lambda_{1}^{+}}\left[c \partial_{x} \lambda_{-j+1}^{+}-\sum_{l=1}^{j} \lambda_{1-l}^{-} \lambda_{-j+l}^{+}\right]
$$

Hence, $\widetilde{\lambda^{+}} \simeq \lambda^{+}$can be approximated as follows

$$
\widetilde{\lambda^{+}}=\sum_{j=1}^{\infty} \lambda_{2-j}^{+}=i \tau+0-\frac{i m^{2} c^{4}}{2} \tau^{-1}+\frac{1}{2 \lambda_{1}^{+}}\left[c \partial_{x} \lambda_{-j+1}^{+}-\sum_{l=1}^{j} \lambda_{1-l}^{-} \lambda_{-j+l}^{+}\right] .
$$

For the sake of conciseness, we limit this discussion to the first three orders ' +1 ', ' 0 ', '-1'. Now by definition 2.1.5, $\Lambda^{+}=\widetilde{\Lambda^{+}}=O p\left(\tilde{\lambda^{+}}\right)$, hence all that is left to do is to revert the symbols back to their operators. We recall that $\sigma\left(\partial_{t}^{-1}\right)=(i \tau)^{-1}=-i \tau^{-1}$, and thus

$$
\widetilde{\Lambda^{+}}=\partial_{t}+\frac{m^{2} c^{4}}{2} \partial_{t}^{-1}
$$

It is now necessary to introduce the Riemann-Liouville integral to define $\partial_{t}^{-1}$ 
Definition 3.1.1 The Riemann-Liouville operator of fractional integration is defined as

$$
D_{t}^{-\nu} f(t)=\frac{1}{\Gamma(\nu)} \int_{0}^{t}(t-\omega)^{\nu-1} f(\omega) \mathrm{d} \omega
$$

for $\nu>0$ where $\Gamma(\nu)$ is the gamma function and $D_{t}^{0} f(t)=f(t)$, assuming that $(t-\cdot)^{\nu-1} f(\cdot)$ is integrable.

So for $\nu=1$

$$
D^{-1} f(t)=\frac{1}{\Gamma(1)} \int_{0}^{t}(t-\omega)^{0} f(w) \mathrm{d} \omega=\int_{0}^{t} f(\omega) \mathrm{d} \omega,
$$

for which (3.39) becomes

$$
\widetilde{\Lambda^{+}} u(x, t)=\partial_{t} u(x, t)+\frac{m^{2} c^{4}}{2} \int_{0}^{t} u(x, \omega) \mathrm{d} \omega .
$$

If $\Omega=[-a, a]$, and $\Gamma=\Gamma_{l} \cup \Gamma_{r}=\{-a\} \cup\{a\}$ then the boundary condition of order ' -1 ', or first precision, for (3.2) at $x=\Gamma_{r}$ is

$$
\left(c \partial_{x}+\widetilde{\Lambda^{+}}\right) u(x, t)=c \partial_{x} u(x, t)+\partial_{t} u(x, t)+\frac{m^{2} c^{4}}{2} \int_{0}^{t} u(x, \omega) \mathrm{d} \omega
$$

Hence our initial boundary value problem with $\mathrm{ABC}$, for the linear laser-free KleinGordon equation is

$$
\left\{\begin{array}{l}
L u(x, t)=\left[-\partial_{t}^{2}+c^{2} \partial_{x}^{2}-m^{2} c^{4}\right] u(x, t)=0, \quad \forall(x, t) \in \Omega \times[0, T], \\
B u(x, t)=c \partial_{x} u(x, t)+\partial_{t} u(x, t)+\frac{m^{2} c^{4}}{2} \int_{0}^{t} u(x, \omega) \mathrm{d} \omega, \quad \forall(x, t) \in \Gamma_{r} \times[0, T], \\
B u(x, t)=c \partial_{x} u(x, t)-\partial_{t} u(x, t)-\frac{m^{2} c^{4}}{2} \int_{0}^{t} u(x, \omega) \mathrm{d} \omega, \quad \forall(x, t) \in \Gamma_{l} \times[0, T], \\
u(x, 0)=\varphi_{0}(x), \quad x \in \Omega
\end{array}\right.
$$

where $u(x, t)=\varphi_{\mid \Omega}(x, t)$ and $\varphi(x, t)$ is the solution of $(3.1)$. 


\subsection{The Klein-Gordon equation with electrody- namic potentials}

We are now ready to tackle a much more applicable version of the problem. In this case, we introduce electrodynamic potentials $A(x, t)$ and $\Phi(x, t)$. These potentials must satisfy Maxwell's equations and $A, \Phi$ are given. The Klein-Gordon equation to solve writes

$$
\left\{\begin{array}{l}
\left(\left(i \hbar \partial_{t}-e \Phi(x, t)\right)^{2}-c^{2}\left(\frac{\hbar}{i} \partial_{x}-e A_{x}(x, t)\right)^{2}-m^{2} c^{4}\right) \varphi=0 \quad \forall(x, t) \in \mathbb{R} \times[0, T] \\
\varphi(x, 0)=\varphi_{0}(x), \quad \forall x \in \mathbb{R} .
\end{array}\right.
$$

As before, $\hbar$ and $c$ are Planck's constant and the speed of light respectively, while $e$ is the charge of a particle, and $m$ is its mass. We refer to [18] for details. After a few manipulations, we obtain

$$
\begin{aligned}
\left(-\hbar^{2} \partial_{t}^{2}+c^{2} \hbar^{2} \partial_{x}^{2}-i e \hbar \Phi \partial_{t}-i c^{2} e \hbar A_{x} \partial_{x}-i e \hbar \partial_{t} \Phi-i c^{2} e \hbar \partial_{x} A_{x}\right. & \\
& \left.+e^{2} \Phi^{2}-c^{2} e^{2} A^{2}-m^{2} c^{4}\right) \varphi=0
\end{aligned}
$$

Let us now introduce a helpful notation, we define the following operators

$$
\begin{aligned}
& O_{a}:=i \hbar e \Phi \partial_{t}, \\
& O_{b}:=i c^{2} \hbar e A_{x} \partial_{x} \\
& O_{d}:=-i \hbar e \partial_{t} \Phi-i c^{2} \hbar e \partial_{x} A_{x}+e^{2} \Phi^{2}-c^{2} e^{2} A_{x}^{2}
\end{aligned}
$$


and the terms preceeding the partial derivatives of our operators are denoted as such

$$
\begin{aligned}
& q_{O_{a}}:=i \hbar e \Phi \\
& q_{O_{b}}:=i c^{2} \hbar e A_{x}, \\
& q_{O_{d}}:=-i \hbar e \partial_{t} \Phi-i c^{2} \hbar e \partial_{x} A_{x}+e^{2} \Phi^{2}-c^{2} e^{2} A_{x}^{2} .
\end{aligned}
$$

We finally choose a form that allows for an easy decomposition

$$
\left(c^{2} \hbar^{2} \partial_{x}^{2}-h^{2} \partial_{t}^{2}-O_{a}-O_{b}+O_{d}-m^{2} c^{4}\right) \varphi=0
$$

We once again introduce a boundary, where $\Omega=[-a, a]$ and $\Gamma=\Gamma_{l} \cup \Gamma_{r}=\{-a\} \cup\{a\}$ just as before, and thus we solve

$$
\left\{\begin{array}{l}
\left(c^{2} \hbar^{2} \partial_{x}^{2}-h^{2} \partial_{t}^{2}-O_{a}-O_{b}+O_{d}-m^{2} c^{4}\right) u(x, t)=0, \quad \forall(x, t) \in \Omega \times[0, T], \\
\left(\partial_{x}+\Lambda\right) u(x, t)=0, \quad(x, t) \in \Gamma \times[0, T] \\
u(x, 0)=\varphi_{0}(x), \quad \forall x \in \Omega
\end{array}\right.
$$

where $\varphi_{0}$ is assumed properly supported in $\Omega$. Let us now apply the technique of factorization and forward bicharacteristics just as was done, hence we factorize our operator, and match the equations such that

$$
\begin{cases}c \hbar\left(\Lambda^{+}-\Lambda^{-}\right) \partial_{x} & =-O_{b}, \\ c \hbar O p\left(\partial_{x} \lambda^{+}\right)-\Lambda^{-} \Lambda^{+} & =-\hbar^{2} \partial_{t}^{2}-q_{O_{a}} \partial_{t}+O_{d}-m^{2} c^{4}\end{cases}
$$

where we use the asymptotic expansion of symbols (3.22), but this time, we limit ourselves to $j=4$

$$
\lambda \simeq \sum_{j=1}^{\infty} \lambda_{2-j}
$$


After some manipulations, we get the following set of equations of equivalence classes

$$
\begin{aligned}
& \left\{\begin{array}{l}
\lambda_{1}^{+}-\lambda_{1}^{-}=0, \\
-\lambda_{1}^{-} \lambda_{1}^{+}=\hbar^{2} \tau^{2},
\end{array}\right. \\
& \begin{cases}c \hbar\left(\lambda_{0}^{+}-\lambda_{0}^{-}\right) & =-q_{O_{b}}, \\
\partial_{x} \lambda_{1}^{+}-\lambda_{1}^{-} \lambda_{0}^{+}-\lambda_{0}^{-} \lambda_{1}^{+}+i \partial_{\tau, \xi} \lambda_{1}^{-} \partial_{t, x} \lambda_{1}^{+} & =-i q_{O_{a}} \tau,\end{cases} \\
& \begin{cases}\lambda_{-1}^{+}-\lambda_{-1}^{-} & =0, \\
\partial_{x} \lambda_{0}^{+}-\lambda_{1}^{-} \lambda_{-1}^{+}-\lambda_{0}^{-} \lambda_{0}^{+}-\lambda_{-1}^{-} \lambda_{1}^{+}, & \\
+i \partial_{\tau, \xi} \lambda_{1}^{-} \partial_{t, x} \lambda_{0}^{+}+i \partial_{\tau, \xi} \lambda_{0}^{-} \partial_{t, x} \lambda_{1}^{+}+\frac{1}{2} \partial_{\tau, \xi}^{2} \lambda_{1}^{-} \partial_{t, x}^{2} \lambda_{1}^{+} & =q_{O_{d}}-m^{2} c^{4},\end{cases} \\
& \begin{cases}\lambda_{-2}^{+}-\lambda_{-2}^{-} & =0 \\
\partial_{x} \lambda_{-1}^{+}-\lambda_{1}^{-} \lambda_{-2}^{+}-\lambda_{0}^{-} \lambda_{-1}^{+}-\lambda_{-1}^{-} \lambda_{0}^{+}-\lambda_{-2}^{-} \lambda_{1}^{+}, & \\
+i \partial_{\tau, \xi} \lambda_{1}^{-} \partial_{t, x} \lambda_{-1}^{+}+i \partial_{\tau, \xi} \lambda_{0}^{-} \partial_{t, x} \lambda_{0}^{+}+i \partial_{\tau, \xi} \lambda_{-1}^{-} \partial_{t, x} \lambda_{1}^{+} & \\
1 & \\
+\frac{1}{2} \partial_{\tau, \xi}^{2} \lambda_{1}^{-} \partial_{t, x}^{2} \lambda_{0}^{+}+\frac{1}{2} \partial_{\tau, \xi}^{2} \lambda_{0}^{-} \partial_{t, x}^{2} \lambda_{1}^{+}-\frac{i}{6} \partial_{\tau, \xi}^{3} \lambda_{1}^{-} \partial_{t, x}^{3} \lambda_{1}^{+}=0\end{cases}
\end{aligned}
$$

It is worth noting that a significant amount of the partial derivative terms are null and $\partial_{\tau, \xi} \lambda^{-}$and $\partial_{t, x} \lambda^{+}$are gradients. Hence from the first set of equations, we obtain the order ' +1 ' condition

$$
\begin{aligned}
& \lambda_{1}^{+}=\lambda_{1}^{-}, \\
& \lambda_{1}^{+}= \pm\left(-\hbar^{2} \tau^{2}\right)^{1 / 2}= \pm i \hbar \tau,
\end{aligned}
$$

and to satisfy (3.6) following a similar analysis to (3.12), we choose $\lambda_{1}^{+}=i \hbar \tau$. Next, after a few manipulations of the second set of equations, we find that

$$
\left\{\begin{aligned}
\lambda_{0}^{+}+\frac{1}{c \hbar} q_{O_{b}} & =\lambda_{0}^{-}, \\
\lambda_{0}^{+} & =\frac{1}{2 \hbar} q_{O_{a}}-\frac{1}{2 c \hbar} q_{O_{b}},
\end{aligned}\right.
$$


where we will denote $q_{O_{e}}=\frac{1}{2 \hbar} q_{O_{a}}-\frac{1}{2 c \hbar} q_{O_{b}}$. We make a small reminder that the order of the symbols on the right must be the same as on the left, hence for $\lambda_{0}^{+}$, the ' 0 ' means that the sum of all the orders of symbols equals exactly ' 0 '. For the next orders, a few more details will be added

$$
\left\{\begin{array}{l}
\lambda_{-1}^{+}=\lambda_{-1}^{-} \\
\lambda_{-1}^{+}=-\frac{1}{2 \lambda_{1}^{+}}\left(q_{O_{d}}+\lambda_{0}^{+} \lambda_{0}^{-}-\partial_{x} \lambda_{0}^{+}-i \partial_{\tau} \lambda_{1}^{-} \partial_{t} \lambda_{0}^{+}-m^{2} c^{4}\right)
\end{array}\right.
$$

and

$$
\left\{\begin{array}{l}
\lambda_{-2}^{+}=\lambda_{-2}^{-} \\
\lambda_{-2}^{+}=-\frac{1}{2 \lambda_{1}^{+}}\left(\lambda_{-1}^{+}\left(\lambda_{0}^{+}+\lambda_{0}^{-}\right)-\partial_{x} \lambda_{-1}^{+}-i \partial_{\tau} \lambda_{1}^{-} \partial_{t} \lambda_{0}^{+}\right)
\end{array}\right.
$$

As we start calculating $\lambda_{-1}^{+}$, we find that

$$
\begin{array}{r}
\lambda_{0}^{+} \lambda_{0}^{-}=\frac{1}{4}\left(\frac{1}{\hbar^{2}} q_{O_{a}}^{2}-\frac{1}{c^{2} \hbar^{2}} q_{O_{b}}^{2}\right) \\
\partial_{x} q_{O_{e}}:=\partial_{x} \lambda_{0}^{+}=\frac{1}{2 \hbar} \partial_{x} q_{O_{a}}-\frac{1}{2 c \hbar} \partial_{x} q_{O_{b}} .
\end{array}
$$

Once again $q_{O_{e}}:=\frac{1}{2 \hbar} q_{O_{a}}-\frac{1}{2 c \hbar} q_{O_{b}}$. After making some manipulations, we obtain

$$
\lambda_{-1}^{+}=\frac{i}{2 \hbar}\left(q_{O_{d}}+\frac{1}{4 \hbar^{2}} q_{O_{a}}^{2}-\frac{1}{4 c^{2} \hbar^{2}} q_{O_{b}}^{2}-\partial_{x} q_{O_{e}}+\hbar \partial_{t} q_{O_{e}}-m^{2} c^{4}\right) \tau^{-1}
$$

as our order ' 1 ' condition for $\lambda_{-1}^{+}$, where we now denote

$$
q_{O_{z}}=\left(q_{O_{d}}+\frac{1}{4 \hbar^{2}} q_{O_{a}}^{2}-\frac{1}{4 c^{2} \hbar^{2}} q_{O_{b}}^{2}-\partial_{x} q_{O_{e}}+\hbar \partial_{t} q_{O_{e}}-m^{2} c^{4}\right)
$$


Lastly, we find that $\lambda_{-2}^{+}$gives

$$
\begin{aligned}
\lambda_{-2}^{+}= & -\frac{1}{2 \lambda_{1}^{+}}\left(2 \lambda_{0}^{+} \lambda_{-1}^{+}+\frac{1}{c \hbar} q_{O_{b}} \lambda_{-1}^{+}-\partial_{x} \lambda_{-1}^{+}-i \partial_{\tau} \lambda_{1}^{-} \partial_{t} \lambda_{-1}^{+}\right) \\
= & \frac{i}{2 \hbar}\left(2\left(\frac{1}{2 \hbar} q_{O_{a}}-\frac{1}{2 c \hbar} q_{O_{b}}\right) \frac{i}{2 \hbar} q_{O_{z}} \tau^{-1}+\frac{1}{c \hbar} q_{O_{b}} \frac{i}{2 \hbar} q_{O_{z}} \tau^{-1}\right. \\
& \left.-\frac{i}{2 \hbar} \partial_{x} q_{O_{z}} \tau^{-1}+\frac{i}{2} \partial_{t} q_{O_{z}} \tau^{-1}\right) \tau^{-1} \\
= & -\frac{1}{4 \hbar}\left(\frac{1}{\hbar} q_{O_{a}} q_{O_{z}}-\frac{1}{\hbar} \partial_{x} q_{O_{z}}+\partial_{t} q_{O_{z}}\right) \tau^{-2}
\end{aligned}
$$

which is the last order we will use for our numerical experiments. Hence the full approximation of the boundary condition operator is given by

$$
\begin{aligned}
\lambda^{+} \simeq \sum_{j=1}^{4} \lambda_{2-j}^{+}= & i \hbar \tau+\frac{1}{2 \hbar} q_{O_{a}}-\frac{1}{2 c \hbar} q_{O_{b}}+\frac{i}{2 \hbar} q_{O_{z}} \tau^{-1} \\
& -\frac{1}{4 \hbar}\left(\frac{1}{\hbar} q_{O_{a}} q_{O_{z}}-\frac{1}{\hbar} \partial_{x} q_{O_{z}}+\partial_{t} q_{O_{z}}\right) \tau^{-2}
\end{aligned}
$$

Now once again, just as was done in previously, we use definition 3.1.1 to revert the symbols back to their operators and obtain the following boundary conditions for $\Gamma_{r}$

$$
\begin{aligned}
& B u(x, t)=\left(c \hbar \partial_{x}+\widetilde{\Lambda^{+}}\right) u(x, t)=0 \\
& =c \hbar \partial_{x} u(x, t)+\hbar \partial_{t} u(x, t)+\frac{1}{2 \hbar} O p\left(q_{O_{a}}\right) u(x, t) \\
& -\frac{1}{2 c \hbar} O p\left(q_{O_{b}}\right) u(x, t)-\frac{1}{2 \hbar} O p\left(q_{O_{z}}\right) \int_{0}^{t} u(x, \omega) \mathrm{d} \omega \\
& +\frac{1}{4 \hbar}\left(\frac{1}{\hbar} O p\left(q_{O_{a}}\right) O p\left(q_{O_{z}}\right)-\frac{1}{\hbar} O p\left(\partial_{x} q_{O_{z}}\right)+O p\left(\partial_{t} q_{O_{z}}\right)\right) \frac{1}{\Gamma(2)} \int_{0}^{t}(t-\omega) u(x, \omega) \mathrm{d} \omega=0
\end{aligned}
$$


hence we solve IBVP with ABC (3.51)

$$
\left\{\begin{array}{l}
L u(x, t)=\left(\left(i \hbar \partial_{t}-q \Phi(x, t)\right)^{2}-c^{2}\left(\frac{\hbar}{i} \partial_{x}-q A_{x}(x, t)\right)^{2}-m^{2} c^{4}\right) u(x, t)=0 \\
\forall(x, t) \in \Omega \times[0, T], \\
B u(x, t)=\left(c \hbar \partial_{x}+\widetilde{\Lambda^{+}}\right) u(x, t)=0, \quad \forall(x, t) \in \Gamma_{r} \times[0, T], \\
B u(x, t)=\left(c \hbar \partial_{x}-\widetilde{\Lambda^{-}}\right) u(x, t)=0, \quad \forall(x, t) \in \Gamma_{l} \times[0, T], \\
u(x, 0)=\varphi_{0}(x), \quad \forall x \in \Omega .
\end{array}\right.
$$

where $u(x, t)=\varphi_{\left.\right|_{\Omega}}(x, t)$ and $\varphi(x, t)$ is the solution of (3.31). Let us conclude by listing the boundary conditions in order.

Proposition 3.2.1 The family of outgoing ABCs for a 1-D, time dependent KleinGordon equation for spinless particles subject to electrodynamic potentials is given by, - leading order ' 1 ':

$$
\widetilde{\Lambda}_{(2,1)}^{+}=\hbar \partial_{t}
$$

Here, the subscript $(2,1)$ means that $m=2$ and $j=1$.

- base order ' 0 ':

$$
\widetilde{\Lambda}_{(2,0)}^{+}=\widetilde{\Lambda}_{(2,1)}^{+}+\frac{1}{2 \hbar} O p\left(q_{O_{a}}\right)-\frac{1}{2 c \hbar} O p\left(q_{O_{b}}\right)
$$

- precision order ' -1 ':

$$
\widetilde{\Lambda}_{(2,-1)}^{+}=\widetilde{\Lambda}_{(2,0)}^{+}-\frac{1}{2 \hbar} O p\left(q_{O_{z}}\right) \partial_{t}^{-1}
$$

- and lastly, precision order '-2':

$$
\widetilde{\Lambda}_{(2,-2)}^{+}=\tilde{\Lambda}_{(2,-1)}^{+}+\frac{1}{4 \hbar}\left(\frac{1}{\hbar} O p\left(q_{O_{a}} q_{O_{z}}\right)-\frac{1}{\hbar} O p\left(\partial_{x} q_{O_{z}}\right)+O p\left(\partial_{t} q_{O_{z}}\right)\right) \partial_{t}^{-2}
$$




\section{Chapter 4}

\section{Klein-Gordon equation in 2-D}

\subsection{The Klein-Gordon equation with electrody- namic potentials}

This section will simply take the same problem as in Section 3.2, however, with two spatial dimensions taken into consideration. It is also subject to electrodynamic potentials $A(x, y, t)$ and $\Phi(x, y, t)$. Then we are to solve the following Klein-Gordon equation

$$
\left\{\begin{array}{l}
{\left[\left(i \hbar \partial_{t}-e \Phi(x, y, t)\right)^{2}-c^{2}\left(\frac{\hbar}{i} \nabla-e A(x, y, t)\right)^{2}-m^{2} c^{4}\right] \varphi(x, y, t),} \\
\forall(x, y) \in \mathbb{R}^{2}, t \in[0, T]=0, \\
\varphi(x, y, 0)=\varphi_{0}(x, y), \quad \forall(x, y) \in \mathbb{R}^{2}, .
\end{array}\right.
$$

Just as Section $3.2, \hbar$ and $c$ are Planck's constant and the speed of light respectively, $e$ is the charge of a particle, and $m$ is its mass. Once again, we refer to [18] for details. 
After few manipulations, the equation expands into

$$
\left\{\begin{aligned}
L u(x, y, t)=[ & {\left[-\hbar^{2} \partial_{t}^{2}+c^{2} \hbar^{2} \Delta-i e \hbar \Phi \partial_{t}-i c^{2} e \hbar A \cdot \nabla-i e \hbar \partial_{t} \Phi\right.} \\
& \left.-i c^{2} e \hbar \nabla \cdot A+e^{2} \Phi^{2}-c^{2} e^{2} A^{2}-m^{2} c^{4}\right] u(x, y, t)=0, \\
& \forall(x, y, t) \in \Omega \times[0, T], \\
B u(x, y, t)= & \left(c \hbar \partial_{x}+\widetilde{\Lambda^{+}}\right) u(x, y, t)=0, \quad \forall(x, y, t) \in \Gamma \times[0, T], \\
u(x, y, 0)= & \varphi_{0}(x, y), \forall(x, y) \in \Omega,
\end{aligned}\right.
$$

where $\varphi_{0}(x, y)$ is assumed properly supported in $\Omega$. Here, we choose a domain $\Omega$ to be a circle with constant radius $r_{c}$, this gives us a constant curvature which simplifies the algebraic computations. Hence the Laplacian at the boundary under local coordinates is $\Delta_{(r, \theta)}=\partial_{r}^{2}+\frac{1}{R} \partial_{r}+\frac{1}{R^{2}} \partial_{\theta}^{2}$ where $R=r_{c}+r$ and $0<r<\epsilon$ as shown in Chapter 2. We note that the Laplacian on arbitrary convex surfaces was determined in [3]. Now in our case, we will also need the first derivatives with respect to $x, y$ in local coordinates, as well as the magnetic field $A(x, y, t)$, so we list them as

$$
\begin{aligned}
& \partial_{x}=\cos (\theta) \partial_{r}-\frac{1}{R} \sin (\theta) \partial_{\theta} \\
& \partial_{y}=\sin (\theta) \partial_{r}+\frac{1}{R} \cos (\theta) \partial_{\theta} \\
& A_{x}(R, \theta)=A_{r} \cos (\theta)-A_{\theta} \sin (\theta) \\
& A_{y}(R, \theta)=A_{r} \sin (\theta)+A_{\theta} \cos (\theta)
\end{aligned}
$$

We now have all the tools necessary to complete the switch to our local coordinates at the boundary $\Gamma=\partial \Omega$.

$$
\begin{aligned}
& {\left[-\hbar^{2} \partial_{t}^{2}+c^{2} \hbar^{2} \partial_{r}^{2}-\frac{1}{R} c^{2} \hbar^{2} \partial_{r}+\frac{1}{R^{2}} c^{2} \hbar^{2} \partial_{\theta}^{2}-i \hbar e \Phi \partial_{t}-i c^{2} \hbar e A_{x} \partial_{x}-i c^{2} \hbar e A_{y} \partial_{y}\right.} \\
& \left.-i \hbar e \partial_{t} \Phi-i c^{2} \hbar e \partial_{x} A_{x}-i c^{2} \hbar e \partial_{y} A_{y}-c^{2} e^{2} A_{r}^{2}-c^{2} e^{2} A_{\theta}^{2}+e^{2} \Phi^{2}-m^{2} c^{4}\right] \varphi=0
\end{aligned}
$$


where $(r, \theta) \in \Gamma$ and $t \in[0, T]$. Let us work out the actual details of $\partial_{x} A_{x}=$ $\left[\cos (\theta) \partial_{r}-\frac{1}{R} \sin (\theta) \partial_{\theta}\right]\left[A_{r} \cos (\theta)-A_{\theta} \sin (\theta)\right]$ and its $\partial_{y} A_{y}, A_{x} \partial_{x}$ and $A_{y} \partial_{y}$ counterparts. The product rule and trigonometric identities become useful here. The same is done for $A^{2}=A_{r}^{2}+A_{\theta}^{2}$. At this point $\partial_{r, s} A_{r, s}(r, s)$ are simply values, and we obtain

$$
\begin{aligned}
& \partial_{x} A_{x}=\left[\cos (\theta) \partial_{r}-\frac{1}{R} \sin (\theta) \partial_{\theta}\right]\left[A_{r} \cos (\theta)-A_{\theta} \sin (\theta)\right], \\
& =\cos (\theta) \partial_{r}\left(A_{r} \cos (\theta)\right)-\cos (\theta) \partial_{r}\left(A_{\theta} \sin (\theta)\right) \\
& -\frac{1}{R} \sin (\theta) \partial_{\theta}\left(A_{r} \cos (\theta)\right)+\frac{1}{R} \sin (\theta) \partial_{\theta} A_{\theta} \sin (\theta), \\
& =\cos ^{2}(\theta) \partial_{r} A_{r}-\cos (\theta) \sin (\theta) \partial_{r} A_{\theta}-\frac{1}{R} \sin (\theta)\left[\partial_{\theta} A_{r} \cos (\theta)+A_{r} \partial_{\theta} \cos (\theta)\right] \\
& +\frac{1}{R} \sin (\theta)\left[\partial_{\theta} A_{\theta} \sin (\theta)+A_{\theta} \partial_{\theta} \sin (\theta)\right], \\
& =\cos ^{2}(\theta) \partial_{r} A_{r}-\cos (\theta) \sin (\theta) \partial_{r} A_{\theta}-\frac{1}{R} \sin (\theta) \cos (\theta) \partial_{\theta} A_{r}+\frac{1}{R} \sin ^{2}(\theta) A_{r} \\
& +\frac{1}{R} \sin ^{2}(\theta) \partial_{\theta} A_{\theta}+\frac{1}{R} \sin (\theta) \cos (\theta) A_{\theta}, \\
& \partial_{y} A_{y}=\left[\sin (\theta) \partial_{r}+\frac{1}{R} \cos (\theta) \partial_{\theta}\right]\left[A_{r} \sin (\theta)+A_{\theta} \cos (\theta)\right], \\
& =\sin (\theta) \partial_{r}\left(A_{r} \sin (\theta)\right)+\sin (\theta) \partial_{r}\left(A_{\theta} \cos (\theta)\right) \\
& +\frac{1}{R} \cos (\theta) \partial_{\theta}\left(A_{r} \sin (\theta)\right)+\frac{1}{R} \cos (\theta) \partial_{\theta}\left(A_{\theta} \cos (\theta)\right), \\
& =\sin ^{2}(\theta) \partial_{r} A_{r}+\sin (\theta) \cos (\theta) \partial_{r} A_{\theta}+\frac{1}{R} \cos (\theta)\left[\partial_{\theta} A_{r} \sin (\theta)+A_{r} \partial_{\theta} \sin (\theta)\right] \\
& +\frac{1}{R} \cos (\theta)\left[\partial_{\theta} A_{\theta} \cos (\theta)+A_{\theta} \partial_{\theta} \cos (\theta)\right] \text {, } \\
& =\sin ^{2}(\theta) \partial_{r} A_{r}-\sin (\theta) \cos (\theta) \partial_{r} A_{\theta}+\frac{1}{R} \cos (\theta) \sin (\theta) \partial_{\theta} A_{r}+\frac{1}{R} \cos ^{2}(\theta) A_{r} \\
& +\frac{1}{R} \cos ^{2}(\theta) \partial_{\theta} A_{\theta}+\frac{1}{R} \cos (\theta) \sin (\theta) A_{\theta},
\end{aligned}
$$


and adding up the terms together gives

$$
\begin{aligned}
\partial_{x} A_{x}+\partial_{y} A_{y}= & \cos ^{2}(\theta) \partial_{r} A_{r}-\cos (\theta) \sin (\theta) \partial_{r} A_{\theta}-\frac{1}{R} \sin (\theta) \cos (\theta) \partial_{\theta} A_{r} \\
& +\frac{1}{R} \sin ^{2}(\theta) A_{r}+\frac{1}{R} \sin ^{2}(\theta) \partial_{\theta} A_{\theta}+\frac{1}{R} \sin (\theta) \cos (\theta) A_{\theta}+\sin ^{2}(\theta) \partial_{r} A_{r} \\
& -\sin (\theta) \cos (\theta) \partial_{r} A_{\theta}+\frac{1}{R} \cos (\theta) \sin (\theta) \partial_{\theta} A_{r}+\frac{1}{R} \cos ^{2}(\theta) A_{r} \\
& +\frac{1}{R} \cos ^{2}(\theta) \partial_{\theta} A_{\theta}+\frac{1}{R} \cos (\theta) \sin (\theta) A_{\theta} \\
= & \partial_{r} A_{r}+\frac{1}{R} A_{r}+\frac{1}{R} \partial_{\theta} A_{\theta}
\end{aligned}
$$

Similarly for $A_{x} \partial_{x}$ and $A_{y} \partial_{y}$ :

$$
\begin{aligned}
A_{x} \partial_{x}= & {\left[A_{r} \cos (\theta)-A_{\theta} \sin (\theta)\right]\left[\cos (\theta) \partial_{r}-\frac{1}{R} \sin (\theta) \partial_{\theta}\right] } \\
= & A_{r} \cos ^{2}(\theta) \partial_{r}-\frac{1}{R} A_{r} \cos (\theta) \sin (\theta) \partial_{\theta} \\
& -A_{\theta} \sin (\theta) \cos (\theta) \partial_{r}+\frac{1}{R} A_{\theta} \sin ^{2}(\theta) \partial_{\theta} \\
A_{y} \partial_{y}= & {\left[A_{r} \sin (\theta)+A_{\theta} \cos (\theta)\right]\left[\sin (\theta) \partial_{r}+\frac{1}{R} \cos (\theta) \partial_{\theta}\right] } \\
= & A_{r} \sin ^{2}(\theta) \partial_{r}+\frac{1}{R} A_{r} \sin (\theta) \cos (\theta) \partial_{\theta} \\
& +A_{\theta} \cos (\theta) \sin (\theta) \partial_{r}+\frac{1}{R} A_{\theta} \cos ^{2}(\theta) \partial_{\theta} \\
A_{x} \partial_{x}+A_{y} \partial_{y}= & A_{r} \cos ^{2}(\theta) \partial_{r}-\frac{1}{R} A_{r} \cos (\theta) \sin (\theta) \partial_{\theta}-A_{\theta} \sin (\theta) \cos (\theta) \partial_{r} \\
& +\frac{1}{R} A_{\theta} \sin ^{2}(\theta) \partial_{\theta}+A_{r} \sin ^{2}(\theta) \partial_{r}+\frac{1}{R} A_{r} \sin (\theta) \cos (\theta) \partial_{\theta} \\
& +A_{\theta} \cos (\theta) \sin (\theta) \partial_{r}+\frac{1}{R} A_{\theta} \cos ^{2}(\theta) \partial_{\theta} \\
A_{x} \partial_{x}+A_{y} \partial_{y}= & A_{r} \partial_{r}+\frac{1}{R} A_{\theta} \partial_{\theta}
\end{aligned}
$$


Hence $\nabla \cdot A$ is given by (4.17) and $A \cdot \nabla$ is given by (4.25). Finally the complete equation in local coordinates at the boundary is

$$
\begin{aligned}
& {\left[-\hbar^{2} \partial_{t}^{2}+c^{2} \hbar^{2} \partial_{r}^{2}-\frac{1}{R} c^{2} \hbar^{2} \partial_{r}+\frac{1}{R^{2}} c^{2} \hbar^{2} \partial_{\theta}^{2}-i \hbar e \Phi \partial_{t}-i c^{2} \hbar e A_{r} \partial_{r}\right.} \\
& -\frac{1}{R} i c^{2} \hbar e A_{\theta} \partial_{\theta}-i \hbar e \partial_{t} \Phi-i c^{2} \hbar e \partial_{r} A_{r}-\frac{1}{R} i c^{2} \hbar e A_{r}-\frac{1}{R} i c^{2} \hbar e \partial_{\theta} A_{\theta} \\
& \left.-c^{2} e^{2} A_{r}^{2}-c^{2} e^{2} A_{\theta}^{2}+e^{2} \Phi^{2}-m^{2} c^{4}\right] \varphi=0
\end{aligned}
$$

Using similar notations as Chapter 3, we define

$$
\begin{aligned}
O_{a} & :=i \hbar e \Phi \partial_{t} \\
O_{b} & :=-\frac{1}{R} c^{2} \hbar^{2} \partial_{r}-i c^{2} \hbar e A_{r} \partial_{r} \\
O_{c} & :=-\frac{1}{R} i c^{2} \hbar e A_{\theta} \partial_{\theta} \\
O_{d} & :=-i \hbar e \partial_{t} \Phi-i c^{2} \hbar e \partial_{r} A_{r}-\frac{1}{R} i c^{2} \hbar e A_{r}-\frac{1}{R} i c^{2} \hbar e \partial_{\theta} A_{\theta}+e^{2} \Phi^{2}-c^{2} e^{2} A^{2}-m^{2} c^{4}
\end{aligned}
$$

and the coefficient of the aforementioned operators are denoted as such

$$
\begin{aligned}
& q_{O_{a}}:=i \hbar e \Phi \\
& q_{O_{b}}:=-\frac{1}{R} c^{2} \hbar^{2}-i c^{2} \hbar e A_{r} \\
& q_{O_{c}}:=-\frac{1}{R} i c^{2} \hbar e A_{\theta} \\
& q_{O_{d}}:=-i \hbar e \partial_{t} \Phi-i c^{2} \hbar e \partial_{r} A_{r}-\frac{1}{R} i c^{2} \hbar e A_{r}-\frac{1}{R} i c^{2} \hbar e \partial_{\theta} A_{\theta}+e^{2} \Phi^{2}-c^{2} e^{2} A^{2}-m^{2} c^{4}
\end{aligned}
$$

We now write things down in a more compact form

$$
\left[c^{2} \hbar^{2} \partial_{r}^{2}-\hbar^{2} \partial_{t}^{2}+\frac{1}{R^{2}} c^{2} \hbar^{2} \partial_{\theta}^{2}-q_{O_{a}} \partial_{t}+q_{O_{b}} \partial_{r}+q_{O_{c}} \partial_{\theta}+q_{O_{d}}\right] \varphi=0
$$


which is ideal for the next step, namely the factorization and decomposition into symbolic equivalence classes, similar to what was previously done in Chapter 3. Hence, by theorem 2.3.1, the principal symbol of $L\left(r, \theta, t, \partial_{r}, \partial_{\theta}, \partial_{t}\right)$ can be factored as follows

$$
\left(c \hbar \partial_{r}-\Lambda^{-}\right)\left(c \hbar \partial_{r}+\Lambda^{+}\right)=c^{2} \hbar^{2} \partial_{r}^{2}+c \hbar\left(\Lambda^{+}-\Lambda^{-}\right) \partial_{r}+c \hbar O p\left(\partial_{r} \lambda^{+}\right)-\Lambda^{-} \Lambda^{+}
$$

and matched to $(4.36)$

$$
\begin{cases}\left(\Lambda^{+}-\Lambda^{-}\right) \partial_{r} & =\frac{1}{c \hbar} q_{O_{b}} \partial_{r} \\ c \hbar O p\left(\partial_{r} \lambda^{+}\right)-\Lambda^{-} \Lambda^{+} & =-\hbar^{2} \partial_{t}^{2}-q_{O_{a}} \partial_{t}+\frac{1}{R^{2}} c^{2} \hbar^{2} \partial_{\theta}^{2}+q_{O_{c}} \partial_{\theta}+O_{d}\end{cases}
$$

This gives the symbols,

$$
\begin{cases}\lambda^{+}-\lambda^{-} & =\frac{1}{c \hbar} q_{O_{b}} \\ c \hbar \partial_{r} \lambda^{+}-\lambda^{-} \# \lambda^{+} & =\hbar^{2} \tau^{2}-i q_{O_{a}} \tau-\frac{1}{R^{2}} c^{2} \hbar^{2} \xi^{2}+i q_{O_{c}} \xi+q_{O_{d}}\end{cases}
$$

Once again, using $\lambda^{ \pm} \simeq \sum_{j=1}^{\infty} \lambda_{2-j}^{ \pm}$, and truncating our asymptotic expansion at $j=4$, we find that our symbolic equations that allow us to explicitly determine the symbols of our operator

$$
\begin{gathered}
\left\{\begin{array}{l}
\lambda_{1}^{+}-\lambda_{1}^{-}=0 \\
-\lambda_{1}^{-} \lambda_{1}^{+}=\hbar^{2} \tau^{2}-\frac{1}{R^{2}} c^{2} \hbar^{2} \xi^{2}
\end{array}\right. \\
\left\{\begin{array}{l}
\lambda_{0}^{+}-\lambda_{0}^{-} \\
c \hbar \partial_{r} \lambda_{1}^{+}-\lambda_{1}^{-} \lambda_{0}^{+}-\lambda_{0}^{-} \lambda_{1}^{+}+i \partial_{\tau, \xi} \lambda_{1}^{-} \partial_{t, x} \lambda_{1}^{+}=\frac{1}{c \hbar} q_{O_{b}}
\end{array}\right.
\end{gathered}
$$




$$
\begin{aligned}
& \begin{cases}\lambda_{-1}^{+}-\lambda_{-1}^{-} & =0, \\
c \hbar \partial_{r} \lambda_{0}^{+}-\lambda_{1}^{-} \lambda_{-1}^{+}-\lambda_{0}^{-} \lambda_{0}^{+}-\lambda_{-1}^{-} \lambda_{1}^{+}, & \\
+i \partial_{\tau, \xi} \lambda_{1}^{-} \partial_{t, x} \lambda_{0}^{+}+i \partial_{\tau, \xi} \lambda_{0}^{-} \partial_{t, x} \lambda_{1}^{+}+\frac{1}{2} \partial_{\tau, \xi}^{2} \lambda_{1}^{-} \partial_{t, x}^{2} \lambda_{1}^{+} & =q_{O_{d}},\end{cases} \\
& \begin{cases}\lambda_{-2}^{+}-\lambda_{-2}^{-} & =0 \\
c \hbar \partial_{r} \lambda_{-1}^{+}-\lambda_{1}^{-} \lambda_{-2}^{+}-\lambda_{0}^{-} \lambda_{-1}^{+}-\lambda_{-1}^{-} \lambda_{0}^{+}-\lambda_{-2}^{-} \lambda_{1}^{+} & \\
+i \partial_{\tau, \xi} \lambda_{1}^{-} \partial_{t, x} \lambda_{-1}^{+}+i \partial_{\tau, \xi} \lambda_{0}^{-} \partial_{t, x} \lambda_{0}^{+}+i \partial_{\tau, \xi} \lambda_{-1}^{-} \partial_{t, x} \lambda_{1}^{+} & \\
1 & \\
+\frac{1}{2} \partial_{\tau, \xi}^{2} \lambda_{1}^{-} \partial_{t, x}^{2} \lambda_{0}^{+}+\frac{1}{2} \partial_{\tau, \xi}^{2} \lambda_{0}^{-} \partial_{t, x}^{2} \lambda_{1}^{+}-\frac{i}{6} \partial_{\tau, \xi}^{3} \lambda_{1}^{-} \partial_{t, x}^{3} \lambda_{1}^{+}= & 0,\end{cases}
\end{aligned}
$$

and we get the leading order term

$$
\left\{\begin{array}{l}
\lambda_{1}^{+}=\lambda_{1}^{-} \\
\lambda_{1}^{+}= \pm\left(-\hbar^{2} \tau^{2}+\frac{c^{2} \hbar^{2}}{R^{2}} \xi^{2}\right)^{1 / 2}
\end{array}\right.
$$

Now, as was done in Chapter 3, we must satisfy (3.6) with an analysis similar to (3.12), $\lambda_{1}^{+}=\left(-\hbar^{2} \tau^{2}+\frac{c^{2} \hbar^{2}}{R^{2}} \xi^{2}\right)^{1 / 2}$ is hence selected as the forward bicharacteristic curve. With the leading order being fairly complicated, and we can already see that trying to find

$$
\lambda_{0}^{+}=-\frac{1}{2 \lambda_{1}^{+}}\left[-i q_{O_{a}} \tau+i q_{O_{c}} \xi-c \hbar \partial_{r} \lambda_{1}^{+}\right]+\frac{1}{2 c \hbar} q_{O_{b}}
$$

is quite the tedious and daunting task. Hence, in practice, it is wise to approximate $\lambda_{1}^{+}$. Theoretically, however, the equations break down as follows: 


$$
\begin{aligned}
\lambda_{1}^{+}= & \left(-\hbar^{2} \tau^{2}+\frac{c^{2} \hbar^{2}}{R^{2}} \xi^{2}\right)^{1 / 2} \\
\lambda_{0}^{+}= & -\frac{1}{2 \lambda_{1}^{+}}\left[-i q_{O_{a}} \tau+i q_{O_{c}} \xi-c \hbar \partial_{r} \lambda_{1}^{+}\right]+\frac{1}{2 c \hbar} q_{O_{b}} \\
\lambda_{0}^{-}= & -\frac{1}{2 \lambda_{1}^{+}}\left[-i q_{O_{a}} \tau+i q_{O_{c}} \xi-c \hbar \partial_{r} \lambda_{1}^{+}\right]-\frac{1}{2 c \hbar} q_{O_{b}} \\
\lambda_{-1}^{+}= & -\frac{1}{2 \lambda_{1}^{+}}\left[q_{O_{d}}-c \hbar \partial_{r} \lambda_{0}^{+}+\lambda_{0}^{+} \lambda_{0}^{-}-i \partial_{\tau} \lambda_{1}^{-} \partial_{t} \lambda_{0}^{+}-i \partial_{\xi} \lambda_{1}^{-} \partial_{\theta} \lambda_{0}^{+}\right] \\
\lambda_{-2}^{+}= & -\frac{1}{2 \lambda_{1}^{+}}\left[\lambda_{-1}^{+}\left[\lambda_{0}^{+}+\lambda_{0}^{-}\right]-c \hbar \partial_{r} \lambda_{-1}^{+}-i \partial_{\tau} \lambda_{1}^{-} \partial_{t} \lambda_{-1}^{+}-i \partial_{\xi} \lambda_{1}^{-} \partial_{\theta} \lambda_{-1}^{+}\right. \\
& \left.-i \partial_{\tau} \lambda_{0}^{-} \partial_{t} \lambda_{0}^{+}-i \partial_{\xi} \lambda_{0}^{-} \partial_{\theta} \lambda_{0}^{+}-\frac{1}{2} \partial_{\xi}^{2} \lambda_{1}^{-} \partial_{\theta}^{2} \lambda_{0}^{+}\right]
\end{aligned}
$$

For the approximation of $\lambda_{1}^{+}$, we first recall that the Taylor expansion of $\sqrt{1+x}=$ $1+\frac{1}{2} x-\frac{1}{8} x^{2}+O\left(x^{3}\right)$ when $|x|<1$. We then use this expansion under high frequencies in time, hence $|\tau| \rightarrow+\infty$ and therefore $\lambda_{1}^{+}$is approximated to

$$
\begin{aligned}
\lambda_{1}^{+} & =\sqrt{-\hbar^{2} \tau^{2}\left(1-\frac{c^{2} \hbar^{2}}{\hbar^{2} R^{2}} \xi^{2} \tau^{-2}\right)} \\
& =i \hbar \tau \sqrt{1-\frac{c^{2}}{R^{2}} \xi^{2} \tau^{-2}} \\
& =i \hbar \tau-\frac{i c^{2} \hbar}{2 R^{2}} \xi^{2} \tau^{-1}+O\left(\tau^{-3}\right)
\end{aligned}
$$

where we truncate at $O\left(\tau^{-3}\right)$. As a general guideline, we note that the sum of the symbols in each term add up to ' +1 ' (i.e. $\xi^{4} \tau^{-3} \Rightarrow 4-3=1$ ), which corresponds to the order ' +1 ' of the operator $\lambda_{1}^{+}$. This will be true for all further calculations. Next we approximate $\lambda_{0}^{+}$, which has a term in $\partial_{r} \lambda_{1}^{+}$and a fractional term $\frac{1}{2 \lambda_{1}^{+}}$. Next, we 
use $\frac{1}{1-x}=1+x+x^{2}+O\left(x^{3}\right)$ when $|x|<1$ to work out the $\frac{1}{2 \lambda_{1}^{+}}$term as

$$
\begin{aligned}
\frac{1}{2 \lambda_{1}^{+}} & =\frac{1}{2 i \hbar \tau-\frac{i c^{2} \hbar}{R^{2}} \xi^{2} \tau^{-1}} \\
& =\left(\frac{1}{2 i \hbar} \tau^{-1}\right)\left(1-\frac{c^{2}}{2 R^{2}} \xi^{2} \tau^{-2}\right)^{-1}=\left(\frac{1}{2 i \hbar} \tau^{-1}\right)\left(1+\frac{c^{2}}{2 R^{2}} \xi^{2} \tau^{-2}+0\left(\tau^{-3}\right)\right)
\end{aligned}
$$

$$
=\frac{1}{2 i \hbar} \tau^{-1}+\frac{c^{2}}{4 i R^{2}} \xi^{2} \tau^{-3}+O\left(\tau^{-5}\right)
$$

and we truncate at $O\left(\tau^{-3}\right)$. We note that $\partial_{r} \frac{1}{\left(r_{c}+r\right)^{2}}=-\frac{2}{R^{3}}$ and thus,

$$
c \hbar \partial_{r} \lambda_{1}^{+}=\frac{i c^{2} \hbar}{R^{3}} \xi^{2} \tau^{-1}+O\left(\tau^{-3}\right)
$$

which gives us all the components we need to complete the appromimation of $\lambda_{0}^{+}$

$$
\begin{aligned}
\lambda_{0}^{+} & =-\frac{1}{2 \lambda_{1}^{+}}\left[-i q_{O_{a}} \tau+i q_{O_{c}} \xi-c \hbar \partial_{r} \lambda_{1}^{+}\right]+\frac{1}{2 c \hbar} q_{O_{b}} \\
& =\left(-\frac{1}{2 i \hbar} \tau^{-1}+O\left(\tau^{-3}\right)\right)\left(-i q_{O_{a}} \tau+i q_{O_{c}} \xi-\frac{i c^{3} \hbar^{2}}{R^{3}} \xi^{2} \tau^{-1}\right)+\frac{1}{2 c \hbar} q_{O_{b}} \\
& =\frac{1}{2 \hbar} q_{O_{a}}+\frac{1}{2 c \hbar} q_{O_{b}}-\frac{1}{2 \hbar} q_{O_{c}} \xi^{-1}+\frac{c^{3} \hbar}{2 R^{3}} \xi^{2} \tau^{-2}+O\left(\tau^{-3}\right) .
\end{aligned}
$$

We note that the sum of orders on each term is ' 0 ', just as desired. It is easy to see that $\lambda_{0}^{-}=\lambda_{0}^{+}-\frac{1}{c \hbar} q_{O_{b}}$, and hence the next step is to now find $\lambda_{-1}^{+}$

$$
\lambda_{-1}^{+}=-\frac{1}{2 \lambda_{1}^{+}}\left[q_{O_{d}}-c \hbar \partial_{r} \lambda_{0}^{+}+\lambda_{0}^{+} \lambda_{0}^{-}-i \partial_{\tau} \lambda_{1}^{-} \partial_{t} \lambda_{0}^{+}-i \partial_{\xi} \lambda_{1}^{-} \partial_{\theta} \lambda_{0}^{+}\right]
$$


We already have the approximation for $\frac{1}{2 \lambda_{1}^{+}}$, which means that we need $c \hbar \partial_{r} \lambda_{0}^{+}$and $\lambda_{0}^{+} \lambda_{0}^{-}$.

$$
\begin{aligned}
& \partial_{r} \lambda_{0}^{+}=\frac{1}{2 \hbar} \partial_{r}\left(q_{O_{a}}\right)+\frac{1}{2 c \hbar} \partial_{r}\left(q_{O_{b}}\right)-\frac{1}{2 \hbar} \partial_{r}\left(q_{O_{c}}\right) \xi \tau^{-1}-\frac{3}{2 r^{4}} c^{3} \hbar^{2} \xi^{2} \tau^{-2}+O\left(\tau^{-3}\right), \\
& \lambda_{0}^{+} \lambda_{0}^{-}=\frac{1}{4 \hbar^{2}} q_{O_{a}}^{2}-\frac{1}{4 c^{2} \hbar^{2}} q_{O_{b}}^{2}-\frac{1}{2 \hbar^{2}} q_{O_{a}} q_{O_{c}} \xi \tau^{-1}+\left(\frac{c^{3}}{2 R^{3}} q_{O_{a}}+\frac{1}{4 \hbar^{2}} q_{O_{c}}^{2}\right) \xi^{2} \tau^{-2}+O\left(\tau^{-3}\right) .
\end{aligned}
$$

We also determine

$$
\begin{aligned}
& i \partial_{\tau} \lambda_{1}^{-} \partial_{t} \lambda_{0}^{+}=-\hbar \partial_{t} q_{O_{g}} \\
& i \partial_{\xi} \lambda_{1}^{-} \partial_{\theta} \lambda_{0}^{+}=\frac{c^{2} \hbar}{R^{2}} \partial_{\theta} q_{O_{h}} \xi \tau^{-1}
\end{aligned}
$$

where $q_{O_{g}}=\left(\frac{1}{2 \hbar} q_{O_{a}}+\frac{1}{2 c \hbar} q_{O_{b}}-\frac{1}{2 \hbar} q_{O_{c}} \xi \tau^{-1}\right)$ and $q_{O_{h}}=\left(\frac{1}{2 \hbar} q_{O_{a}}+\frac{1}{2 c \hbar} q_{O_{b}}\right)$ truncated at $O\left(\tau^{-3}\right)$. Now, once again, we have everything we need to put $\lambda_{-1}^{+}$together and we obtain

$$
\begin{aligned}
\lambda_{-1}^{+}= & \left(-\frac{1}{2 i \hbar} \tau^{-1}\right)\left(q_{O_{d}}-\frac{1}{2} c \partial_{r} q_{O_{a}}-\frac{1}{2} \partial_{r} q_{O_{b}}+\frac{1}{4 \hbar^{2}} q_{O_{a}}^{2}-\frac{1}{4 c^{2} \hbar^{2}} q_{O_{b}}^{2}+\hbar \partial_{t} q_{O_{g}}\right. \\
& \left.-\frac{1}{2 \hbar^{2}} q_{O_{a}} q_{O_{c}} \xi \tau^{-1}+\frac{1}{2} c \partial_{r} q_{O_{c}} \xi \tau^{-1}-\frac{c^{2} \hbar}{R^{2}} \partial_{\theta} q_{O_{h}} \xi \tau^{-1}+O\left(\tau^{-2}\right)\right)
\end{aligned}
$$


Thanks to the properties of symbols, truncating at $O\left(\tau^{-3}\right)$ allows for an accurate computation and $\lambda_{-1}^{+}$can finally be developped as

$$
\begin{aligned}
\lambda_{-1}^{+}= & \left(\frac{i}{2 \hbar} q_{O_{d}}-\frac{i c}{4 \hbar} \partial_{r} q_{O_{a}}-\frac{i}{4 \hbar} \partial_{r} q_{O_{b}}+\frac{i}{8 \hbar^{3}} q_{O_{a}}^{2}-\frac{i}{8 c^{2} \hbar^{3}} q_{O_{b}}^{2}+\hbar \partial_{t} q_{O_{h}}\right) \tau^{-1} \\
& +\left(\frac{i c}{4 \hbar} \partial_{r} q_{O_{c}}-\frac{i}{4 \hbar^{3}} q_{O_{a}} q_{O_{c}}-\frac{c^{2} \hbar}{R^{2}} \partial_{\theta} q_{O_{h}}-\frac{i}{4} \partial_{t} q_{O_{c}}\right) \xi \tau^{-2}+O\left(\tau^{-3}\right)
\end{aligned}
$$

We denote the terms of $\lambda_{-1}^{+}$by

$$
\begin{aligned}
q_{O_{z}} & =\left(\frac{i}{2 \hbar} q_{O_{d}}-\frac{i c}{4 \hbar} \partial_{r} q_{O_{a}}-\frac{i}{4 \hbar} \partial_{r} q_{O_{b}}+\frac{i}{8 \hbar^{3}} q_{O_{a}}^{2}-\frac{i}{8 c^{2} \hbar^{3}} q_{O_{b}}^{2}+\hbar \partial_{t} q_{O_{h}}\right) \\
q_{O_{z z}} & =\left(\frac{i c}{4 \hbar} \partial_{r} q_{O_{c}}-\frac{i}{4 \hbar^{3}} q_{O_{a}} q_{O_{c}}-\frac{c^{2} \hbar}{R^{2}} \partial_{\theta} q_{O_{h}}-\frac{i}{4} \partial_{t} q_{O_{c}}\right)
\end{aligned}
$$

The calculation becomes fairly trivial afterwards as we can exclude the terms that will exceed $O\left(\tau^{-3}\right)$. So last but not least, we work out our last term

$$
\begin{gathered}
\lambda_{-2}^{+}=-\frac{1}{2 \lambda_{1}^{+}}\left[\lambda_{-1}^{+}\left[\lambda_{0}^{+}+\lambda_{0}^{-}\right]-c \hbar \partial_{r} \lambda_{-1}^{+}-i \partial_{\tau} \lambda_{1}^{-} \partial_{t} \lambda_{-1}^{+}-i \partial_{\xi} \lambda_{1}^{-} \partial_{\theta} \lambda_{-1}^{+}\right. \\
\left.-i \partial_{\tau} \lambda_{0}^{-} \partial_{t} \lambda_{0}^{+}-i \partial_{\xi} \lambda_{0}^{-} \partial_{\theta} \lambda_{0}^{+}-\frac{1}{2} \partial_{\xi}^{2} \lambda_{1}^{-} \partial_{\theta}^{2} \lambda_{0}^{+}\right]
\end{gathered}
$$

and can quickly deduce that $\lambda_{0}^{+}+\lambda_{0}^{-}$is simply equal to $2 \lambda_{0}^{+}-\frac{1}{c \hbar} q_{O_{b}}$, and by truncating at $O\left(\tau^{-3}\right)$, the equation develops into

$$
\begin{aligned}
\lambda_{-2}^{+}=\left(-\frac{1}{2 i \hbar} \tau^{-1}\right) & {\left[\left(\frac{1}{\hbar} q_{O_{a}}+\frac{1}{c \hbar} q_{O_{b}}\right) q_{O_{z}} \tau^{-1}-\frac{1}{c \hbar} q_{O_{b}} q_{O_{z}} \tau^{-1}-c \hbar \partial_{r} q_{O_{z}} \tau^{-1}\right.} \\
& \left.+\hbar \partial_{t} q_{O_{z}} \tau^{-1}+\frac{i}{2 \hbar} q_{O_{c}} \partial_{\theta} q_{O_{h}} \tau^{-1}+\frac{i c^{2} \hbar}{2 R^{2}} \partial_{\theta}^{2} q_{O_{h}} \tau^{-1}\right]
\end{aligned}
$$


which then gives

$$
\lambda_{-2}^{+}=\left(\frac{i}{2 \hbar^{2}} q_{O_{a}} q_{O_{z}}-\frac{i c}{2} \partial_{r} q_{O_{z}}+\frac{i}{2} \partial_{t} q_{O_{z}}-\frac{1}{4 \hbar^{2}} q_{O_{c}} \partial_{\theta} q_{O_{h}}-\frac{c^{2}}{4 R^{2}} \partial_{t}^{2} q_{O_{h}}\right) \tau^{-2}+O\left(\tau^{-3}\right)
$$

We are now in position to write out the whole approximate sum for large $\tau$

$$
\begin{aligned}
& \sum_{j=1}^{4} \lambda_{2-j}^{+}= i \hbar \tau \\
&+\frac{1}{2 \hbar} q_{O_{a}}-\frac{1}{2 \hbar} q_{O_{c}} \xi \tau^{-1}+\frac{c^{3} \hbar}{2 R^{3}} \xi^{2} \tau^{-2}+\frac{1}{2 c \hbar} q_{O_{b}}+q_{O_{z}} \tau^{-1}+q_{O_{z z}} \tau^{-2} \\
&+\left(\frac{i}{2 \hbar^{2}} q_{O_{a}} q_{O_{z}}-\frac{i c}{2} \partial_{r} q_{O_{z}}+\frac{i}{2} \partial_{t} q_{O_{z}}\right. \\
&\left.-\frac{1}{4 \hbar^{2}} q_{O_{c}} \partial_{\theta} q_{O_{h}}-\frac{c^{2}}{4 R^{2}} \partial_{t}^{2} q_{O_{h}}\right) \tau^{-2}+O\left(\tau^{-3}\right)
\end{aligned}
$$

and after a few manipulations

$$
\begin{aligned}
& \sum_{j=1}^{4} \lambda_{2-j}^{+}= i \hbar \tau \\
&+\frac{1}{2 \hbar} q_{O_{a}}+\frac{1}{2 c \hbar} q_{O_{b}}+\left(-\frac{1}{2 \hbar} q_{O_{c}} \xi+q_{O_{z}}\right) \tau^{-1} \\
&+\left(\frac{c^{3} \hbar}{2 R^{3}} \xi^{2}+q_{O_{z z}}+\frac{i}{2 \hbar^{2}} q_{O_{a}} q_{O_{z}}-\frac{i c}{2} \partial_{r} q_{O_{z}}+\frac{i}{2} \partial_{t} q_{O_{z}}\right. \\
&\left.-\frac{1}{4 \hbar^{2}} q_{O_{c}} \partial_{\theta} q_{O_{h}}-\frac{c^{2}}{4 R^{2}} \partial_{t}^{2} q_{O_{h}}\right) \tau^{-2}+O\left(\tau^{-3}\right)
\end{aligned}
$$

We keep working with the more compact version and, as was done in Chapter 3, we revert back to the operators noting that $\sigma\left(\partial_{t}^{-1}\right)=(i \tau)^{-1}=-i \tau^{-1}$ and $\partial_{t}^{-2}=-\tau^{-2}$

$$
\begin{aligned}
\widetilde{\Lambda}_{(2, k)}^{+}= & \hbar \partial_{t}+\frac{1}{2 \hbar} O p\left(q_{O_{a}}\right)+\frac{1}{2 c \hbar} O p\left(q_{O_{b}}\right)+i\left(-\frac{i}{2 \hbar} O p\left(q_{O_{c}}\right) \partial_{\theta}+O p\left(q_{O_{z}}\right)\right) \partial_{t}^{-1} \\
& -\left(-\frac{c^{3} \hbar}{2 R^{3}} \partial_{\theta}^{2}+O p\left(q_{O_{z z}}\right)+\frac{i}{2 \hbar^{2}} O p\left(q_{O_{a}}\right) O p\left(q_{O_{z}}\right)-\frac{i c}{2} \partial_{r} O p\left(q_{O_{z}}\right)+\frac{i}{2} \partial_{t} O p\left(q_{O_{z}}\right)\right. \\
& \left.-\frac{1}{4 \hbar^{2}} O p\left(q_{O_{c}}\right) \partial_{\theta} O p\left(q_{O_{h}}\right)-\frac{c^{2}}{4 R^{2}} \partial_{t}^{2} O p\left(q_{O_{h}}\right)\right) \partial_{t}^{-2}+O\left(\partial_{t}^{-3}\right)
\end{aligned}
$$


Proposition 4.1.1 The family of outgoing ABCs for a 2-D, time dependent KleinGordon equation is given by

$$
\left(c \hbar \partial_{r}+\widetilde{\Lambda}_{(2, k)}^{+}\right) \varphi(r, \theta, t)=0
$$

for $k=1,0,-1,-2 .$, where

- leading order ' 1 ':

$$
\widetilde{\Lambda}_{(2,1)}^{+}=\hbar \partial_{t}
$$

- base order ' 0 ':

$$
\widetilde{\Lambda}_{(2,0)}^{+}=\widetilde{\Lambda}_{(2,1)}^{+}+\frac{1}{2 \hbar} O p\left(q_{O_{a}}\right)+\frac{1}{2 c \hbar} O p\left(q_{O_{b}}\right),
$$

- precision order '-1':

$$
\tilde{\Lambda}_{(2,-1)}^{+}=\tilde{\Lambda}_{(2,0)}^{+}+\left(\frac{1}{2 \hbar} O p\left(q_{O_{c}}\right) \partial_{\theta}+O p\left(q_{O_{z}}\right)\right) \partial_{t}^{-1}
$$

- Lastly, precision order '-2':

$$
\begin{aligned}
\widetilde{\Lambda}_{(2,-2)}^{+}=\widetilde{\Lambda}_{(2,-1)}^{+}- & \left(-\frac{c^{3} \hbar}{2 R^{3}} \partial_{\theta}^{2}+O p\left(q_{O_{z z}}\right)+\frac{i}{2 \hbar^{2}} O p\left(q_{O_{a}}\right) O p\left(q_{O_{z}}\right)-\frac{i c}{2} O p\left(\partial_{r} q_{O_{z}}\right)\right. \\
& \left.+\frac{i}{2} O p\left(\partial_{t} q_{O_{z}}\right),-\frac{1}{4 \hbar^{2}} O p\left(q_{O_{c}}\right) O p\left(\partial_{\theta} q_{O_{h}}\right)-\frac{c^{2}}{4 R^{2}} O p\left(\partial_{t}^{2} q_{O_{h}}\right)\right) \partial_{t}^{-2}
\end{aligned}
$$

Now, once again, using definition 3.1.1 in order to calculate the negative differential terms,

$$
\begin{aligned}
& D^{-1} f(t)=\frac{1}{\Gamma(1)} \int_{0}^{t}(t-\omega)^{0} f(w) \mathrm{d} \omega=\int_{0}^{t} f(\omega) \mathrm{d} \omega \\
& D^{-2} f(t)=\frac{1}{\Gamma(2)} \int_{0}^{t}(t-\omega)^{1} f(\omega) \mathrm{d} \omega=\int_{0}^{t}(t-\omega) f(\omega) \mathrm{d} \omega
\end{aligned}
$$


and thus we obtain

$$
\begin{aligned}
\widetilde{\Lambda}_{(2,-2)}^{+} u(r, \theta, t)= & \hbar \partial_{t} u(r, \theta, t)+\frac{1}{2 \hbar} q_{O_{a}} u(r, \theta, t)+\frac{1}{2 c \hbar} q_{O_{b}} u(r, \theta, t) \\
& +i\left(\frac{i}{2 \hbar} q_{O_{c}} \partial_{\theta}+q_{O_{z}}\right) \int_{0}^{t} u(r, \theta, \omega) \mathrm{d} \omega \\
& -\left(-\frac{c^{3} \hbar}{2 R^{3}} \partial_{\theta}^{2}+q_{O_{z z}}+\frac{i}{2 \hbar^{2}} q_{O_{a}} q_{O_{z}}-\frac{i c}{2} \partial_{r} q_{O_{z}}+\frac{i}{2} \partial_{t} q_{O_{z}}\right. \\
& \left.-\frac{1}{4 \hbar^{2}} q_{O_{c}} \partial_{\theta} q_{O_{h}}-\frac{c^{2}}{4 R^{2}} \partial_{t}^{2} q_{O_{h}}\right) \int_{0}^{t}(t-\omega) u(r, \theta, \omega) \mathrm{d} \omega,
\end{aligned}
$$

where for conciseness, we allow a slight abuse of notation $q_{O_{a}}=O p\left(q_{O_{a}}\right)$. Finally the boundary condition of (4.35) is

$$
B u(r, \theta, t)=\left(c \hbar \partial_{r}+\widetilde{\Lambda}_{(2,-2)}^{+}\right) u(r, \theta, t)=0,
$$

which gives us the following boundary value problem

$$
\left\{\begin{aligned}
L u(r, \theta, t)= & \left(c^{2} \hbar^{2} \partial_{r}^{2}-\hbar^{2} \partial_{t}^{2}+\frac{1}{R^{2}} c^{2} \hbar^{2} \partial_{\theta}^{2}-q_{O_{a}} \partial_{t}\right. \\
& \left.+q_{O_{b}} \partial_{r}+q_{O_{c}} \partial_{\theta}+q_{O_{d}}\right) u(r, \theta, t)=0, \forall(r, \theta, t) \in \Omega \times[0, T], \\
B u(r, \theta, t)= & \left(c \hbar \partial_{r}+\widetilde{\Lambda^{+}}(2,-2)\right) u(r, \theta, t)=0, \forall(r, \theta, t) \in \Gamma \times[0, T], \\
u(r, \theta, 0)= & \varphi_{0}(r, \theta), \quad \forall(r, \theta) \in \Omega .
\end{aligned}\right.
$$




\section{Chapter 5}

\section{Numerical analysis}

In this Chapter, we will approximate the Klein-Gordon equation whose particles are subject to electrodynamic potentials with the ABCs derived in Section 3.2. In the first part, we will aim to demonstrate that the choice of schemes will lead to a convergent numerical solution. In order to do that, we shall start by introducing some notations of our finite difference schemes and continue with the study the consistency and stability of the numerical schemes for the 1-D laser-free Klein-Gordon equation. Furthermore, we outline important theorems referenced in [19], particularly the LaxRichtmyer equivalence theorem, which asserts that a scheme is convergent if it is consistent and stable.

In the second part, we will present the results of the Klein-Gordon equation subject to a given electric field. We shall use the schemes studied in the previous section, and apply them directly to

$$
\left\{\begin{array}{l}
\left(\left(i \hbar \partial_{t}-e \phi(x, t)\right)^{2}-c^{2}\left(\frac{\hbar}{i} \partial_{x}-e A_{x}(x, t)\right)^{2}-m^{2} c^{4}\right) \varphi=0 \quad \forall(x, t) \in \Omega \times[0, T] \\
\varphi(x, 0)=\varphi_{0}(x), \quad \forall x \in \Omega .
\end{array}\right.
$$

Finally, we then compare the effectiveness of different boundary conditions of proposition 3.2.1, by comparing the logarithmic reflection amplitude. 


\subsection{Finite difference analysis}

In this section, for the sake of simplicity we study the stability of the laser-free KleinGordon equation, even though the results in the next section will be for the case with laser. We begin by recalling the laser-free Klein-Gordon equation in Chapter 3

$$
\left\{\begin{array}{l}
P \varphi(x, t)=\left[-\partial_{t}^{2}+c^{2} \partial_{x}-m^{2} c^{4}\right] \varphi(x, t)=0, \quad(x, t) \in \Omega \times[0, T] \\
\varphi(x, 0)=\varphi_{0}(x), \quad x \in \Omega
\end{array}\right.
$$

where $\Omega=[-10,10]$. To discuss the finite difference schemes, we start by defining our grid points in $t$ and $x$. Let $\Delta x$ and $\Delta t$ be the space and time step, respectively, then the grid of points will be $\left(x_{j}, t_{n}\right)=(j \Delta x, n \Delta t)$ for arbitrary integers $n$ and $j$. We note that $\Delta t$ is a constant time step. The notation $u_{j}^{n}$ is the numerical approximation of $\varphi(x, t)$, at the grid points $\left(x_{j}, t_{n}\right)$. The idea here is to replace the derivatives by a finite difference scheme, for example

$$
\frac{\partial u\left(x_{j}, t_{n}\right)}{\partial t} \simeq \frac{u\left(x_{j}, t_{n}+\Delta t\right)-u\left(x_{j}, t_{n}\right)}{\Delta t} .
$$

Here we apply a second order centered difference scheme as described by [19], to which we refer to for more detail. Hence equation (5.1) becomes

$$
u_{j}^{n+1}-2 u_{j}^{n}+u_{j}^{n-1}=c^{2} \frac{\Delta t^{2}}{\Delta x^{2}}\left(u_{j+1}^{n}-2 u_{j}^{n}+u_{j-1}^{n}\right)-m^{2} c^{4} u_{j}^{n} .
$$

To study the convergence of our scheme, we first note the Lax-Richtmyer equivalence theorem shown in [19]:

A consistent finite difference scheme, for a partial differential equation for which the initial value problem is well-posed, is convergent if and only if it is stable. This implies that we must study both the consistency and stability of our equation. Hence let us define and show the consistency of our scheme:

Given a partial differential equation, $P \varphi=0$, and a finite difference scheme, 
$P_{\Delta t, \Delta x} u=0$, we say that the finite difference scheme is consistent with the partial differential equation if for any smooth function $\phi(x, t)$

$$
P \phi-P_{\Delta t, \Delta x} \phi \rightarrow 0
$$

as $\Delta t$ and $\Delta x$ tend to 0 . The convergence here is pointwise convergent at each $(x, t)$.

For more detail, we once again refer to [19]. Applying this definition to (5.4), we obtain

$$
P \phi_{\Delta t, \Delta x}=\frac{c^{2}}{\Delta x^{2}}\left(\phi_{j+1}^{n}-2 \phi_{j}^{n}+\phi_{j-1}^{n}\right)-\frac{1}{\Delta t^{2}}\left(\phi_{j}^{n+1}-2 \phi_{j}^{n}+\phi_{j}^{n-1}\right)-m^{2} c^{4} \phi_{j}^{n}=0
$$

where we use the Taylor series of the function $\phi_{j}^{n}=\phi\left(x_{j}, t_{n}\right)$ about $t$ and $x$,

$$
\begin{aligned}
& \phi_{j}^{n+1}=\phi_{j}^{n}+\Delta t\left(\phi_{t}\right)_{j}^{n}+\frac{1}{2} \Delta t^{2}\left(\phi_{t t}\right)_{j}^{n}+O\left(\Delta t^{3}\right)+O\left(\Delta t^{4}\right), \\
& \phi_{j}^{n-1}=\phi_{j}^{n}-\Delta t\left(\phi_{t}\right)_{j}^{n}+\frac{1}{2} \Delta t^{2}\left(\phi_{t t}\right)_{j}^{n}-O\left(\Delta t^{3}\right)+O\left(\Delta t^{4}\right), \\
& \phi_{j+1}^{n}=\phi_{j}^{n}+\Delta x\left(\phi_{x}\right)_{j}^{n}+\frac{1}{2} \Delta x^{2}\left(\phi_{x x}\right)_{j}^{n}+O\left(\Delta x^{3}\right)+O\left(\Delta t^{4}\right), \\
& \phi_{j-1}^{n}=\phi_{j}^{n}-\Delta x\left(\phi_{x}\right)_{j}^{n}+\frac{1}{2} \Delta x^{2}\left(\phi_{x x}\right)_{j}^{n}-O\left(\Delta x^{3}\right)+O\left(\Delta t^{4}\right),
\end{aligned}
$$

which we now include into (5.6):

$$
P \phi_{\Delta t, \Delta x}=\frac{c^{2}}{\Delta x^{2}}\left(\Delta x^{2}\left(\phi_{x x}\right)_{j}^{n}+O\left(\Delta x^{4}\right)\right)-\frac{1}{\Delta t^{2}}\left(\Delta t^{2}\left(\phi_{t t}\right)_{j}^{n}+O\left(\Delta t^{4}\right)\right)-m^{2} c^{4} \phi_{j}^{n}
$$


Now we show that our scheme is consistent

$$
\begin{aligned}
P \phi-P \phi_{\Delta t, \Delta x}= & c^{2}\left(\phi_{x x}\right)_{j}^{n}-\left(\phi_{t t}\right)_{j}^{n}-m^{2} c^{4} \phi_{j}^{n} \\
& -c^{2}\left(\phi_{x x}\right)_{j}^{n}+\left(\phi_{t t}\right)_{j}^{n}+m^{2} c^{4} \phi_{j}^{n}+O\left(\Delta t^{2}\right)+O\left(\Delta x^{2}\right) \rightarrow 0
\end{aligned}
$$

as $\Delta x, \Delta t \rightarrow 0$, then one can easily verify that (5.12) indeed tends towards 0 and hence the scheme is consistent.

We now introduce the discrete Fourier transform

$$
\hat{u}^{n}(\xi)=\frac{\Delta x}{\sqrt{2 \pi}} \sum_{j=-\infty}^{\infty} e^{-i \Delta x \xi} u_{j}^{n}
$$

and we will show that using the Fourier inversion formula,

$$
u_{j}^{n}(x)=\frac{1}{\sqrt{2 \pi}} \int_{-\pi / \Delta x}^{\pi / \Delta x} e^{i j \Delta x \xi} \hat{u}^{n}(\xi) \mathrm{d} \xi
$$

and consequently

$$
\begin{aligned}
u_{j+1}^{n}(x) & =\frac{1}{\sqrt{2 \pi}} \int_{-\pi / \Delta x}^{\pi / \Delta x} e^{i j \Delta x \xi} e^{i \Delta x \xi} \hat{u}^{n}(\xi) \mathrm{d} \xi \\
u_{j-1}^{n}(x) & =\frac{1}{\sqrt{2 \pi}} \int_{-\pi / \Delta x}^{\pi / \Delta x} e^{i j \Delta x \xi} e^{-i \Delta x \xi} \hat{u}^{n}(\xi) \mathrm{d} \xi
\end{aligned}
$$

one can determine an amplification factor to study the stability of our scheme. Now if we apply (5.15) and (5.16) to (5.4), we obtain

$$
\hat{u}^{n+1}(\xi)-2 \hat{u}^{n}(\xi)+\hat{u}^{n-1}(\xi)=c^{2} \nu^{2}\left(e^{i \Delta x \xi}-2+e^{-i \Delta x \xi}\right) \hat{u}^{n}(\xi)-\Delta t^{2} m^{2} c^{4} \hat{u}^{n}(\xi)
$$

where $\nu=\Delta t / \Delta x$.

Let $\hat{u}^{n}(\xi)=g(\Delta x \xi) \hat{u}^{0}(\xi)$ and let $\theta=\Delta x \xi$ for $\theta \in[-\pi / \Delta x, \pi / \Delta x]$, then $(5.17)$ 
becomes

$$
\begin{aligned}
& g(\theta)-2+g(\theta)^{-1}=c^{2} \nu^{2}(2 \cos (\theta)-2)-\Delta t^{2} m^{2} c^{4}, \\
& g(\theta)^{2}-2 g(\theta)+1=2 c^{2} \nu^{2}(\cos (\theta)-1) g(\theta)-\Delta t^{2} m^{2} c^{4} g(\theta),
\end{aligned}
$$

We must now introduce the stability condition:

A finite difference scheme $P_{\Delta t, \Delta x} u=0$ for an equation that is second order in $t$ is conditionally stable, if there is an integer $K$ and for any positive time $T$ there is a constant $C_{T}$ such that

$$
\Delta x \sum_{j=-\infty}^{\infty}\left|u_{j}^{n}\right|^{2} \leq\left(1+n^{2}\right) C_{T} \Delta x \sum_{k=0}^{K} \sum_{j=-\infty}^{\infty}\left|u_{j}^{k}\right|^{2}
$$

for all solutions $u_{j}^{n}$ and for $0 \leq n \Delta t \leq T$. Again, we refer to [19] for details. We also place conditions on the amplification factor:

For a second order time-dependent equation with amplification factor $g(\theta)$ independent of $\Delta t$ and $\Delta x$, the necessary and sufficient condition for the finite difference scheme to be stable is that all roots of $g(\theta)$ satisfy the following conditions:

- $|g(\theta)| \leq 1$

- $|g(\theta)|=1$, then $g(\theta)$ has at most a double root.

Now from (5.19), we evaluate the stability

$$
\begin{array}{r}
g^{2}-2\left(1-2 c^{2} \nu^{2} \sin ^{2}\left(\frac{\theta}{2}\right) g-\Delta t^{2} \frac{m^{2} c^{4}}{2}\right)+1=0 \\
g^{2}-2(1-\beta) g+1=0
\end{array}
$$


where $\beta=2 c^{2} \nu^{2} \sin ^{2}(\theta / 2)+\Delta t^{2} m^{2} c^{4} / 2$. Then we can say that

$$
\left\{\begin{array}{l}
g_{1} g_{2}=1, \\
g_{1}+g_{2}=2(1-\beta),
\end{array}\right.
$$

and thus we can evaluate the discriminant $\Delta(\theta)=-4 \beta(2-\beta)$. When $\Delta(\theta)>0, \forall \theta$, we obtain two real and distinct roots, $\left|g_{1} g_{2}\right|=1$. Hence if $\left|g_{1}\right|<1$ then $\left|g_{2}\right|>1$, and vice-versa, therefore our scheme is unstable when $\Delta(\theta)>0$.

When $\Delta(\theta) \leq 0$, then $\forall \theta$

$$
\begin{aligned}
-4(2-\beta) & \leq 0, \\
2-\beta & \geq 0, \\
\beta & \leq 2, \\
c^{2} \nu^{2} \sin ^{2}\left(\frac{\theta}{2}\right)+\Delta t^{2} \frac{m^{2} c^{4}}{4} & \leq 1,
\end{aligned}
$$

since $\beta>0$. Taking the maximum value of $\sin ^{2}\left(\frac{\theta}{2}\right)$ then we obtain

$$
\begin{array}{r}
c^{2} \frac{\Delta t^{2}}{\Delta x^{2}}\left(1+\frac{m^{2} c^{2}}{4} \Delta x^{2}\right) \leq 1, \\
\Delta t \leq \frac{\Delta x}{c \sqrt{1+\frac{m^{2} c^{2}}{4} \Delta x^{2}}},
\end{array}
$$

which is a condition for the stability of our scheme. In practice, this stability condition is somewhat restrictive and a simple alternative would be to use an implicit or semiimplicit scheme for the mass term $m^{2} c^{4} u$, which allows for more flexibility. Which is used in the next section. 


\subsection{Numerical results}

The discretization that has been discussed and that will be used are fairly primitive, however, they have for purpose to test the functioning of our ABCs. We recall the Klein-Gordon equation discussed in Section 3.2,

$$
P \varphi(x, t)=\left(c^{2} \hbar^{2} \partial_{x}^{2}-h^{2} \partial_{t}^{2}-O_{a}-O_{b}+O_{d}-m^{2} c^{4}\right) \varphi(x, t)=0
$$

where

$$
\begin{aligned}
& O_{a}=i \hbar e \Phi \partial_{t} \\
& O_{b}=i c^{2} \hbar e A_{x} \partial_{x} \\
& O_{d}=-i \hbar e \partial_{t} \Phi-i c^{2} \hbar e \partial_{x} A_{x}+e^{2} \Phi^{2}-c^{2} e^{2} A_{x}^{2}
\end{aligned}
$$

and

$$
\begin{aligned}
& q_{O_{a}}=i \hbar e \Phi \\
& q_{O_{b}}=i c^{2} \hbar e A_{x} \\
& q_{O_{d}}=-i \hbar e \partial_{t} \Phi-i c^{2} \hbar e \partial_{x} A_{x}+e^{2} \Phi^{2}-c^{2} e^{2} A_{x}^{2} .
\end{aligned}
$$

We choose the initial data to be given by a simple Gaussian wave packet, the same as $[10]$

$$
u_{0}(x)=10 e^{-\frac{1}{4} x^{2}} e^{i x k_{0}}
$$

where the wave number is fixed to $k_{0}=5$. We consider this semi-implicit scheme,

$$
\begin{aligned}
& \frac{c^{2} \hbar^{2}}{\Delta x^{2}}\left(u_{j+1}^{n}-2 u_{j}^{n}+u_{j-1}^{n}\right)-\frac{\hbar^{2}}{\Delta t^{2}}\left(u_{j}^{n+1}-2 u_{j}^{n}+u_{j}^{n-1}\right)-\frac{1}{\Delta t}\left(q_{O_{a}}\right)_{j}^{n}\left(u_{j}^{n}-u_{j}^{n-1}\right) \\
& -\left(q_{O_{b}}\right)_{j}^{n} \frac{1}{2 \Delta x}\left(u_{j+1}^{n}-u_{j-1}^{n}\right)+\frac{1}{2}\left(q_{O_{d}}\right)_{j}^{n}\left(u_{j}^{n+1}+u_{j}^{n}\right)-\frac{m^{2} c^{4}}{2}\left(u_{j}^{n+1}+u_{j}^{n}\right)=0,
\end{aligned}
$$


which is a natural extension of the laser-free scheme on the bounded domain $\Omega=$ $[-10,10]$. We also impose the following $\hbar=c=m=e=1$. Now, at the boundaries $x_{l}=-10$ and $x_{r}=10$, we will use the ABCs derived in Section 3.2, proposition 3.2.1, to compare them to the Dirichlet data. We recall

- leading order ' 1 ':

$$
\tilde{\Lambda}_{(2,1)}^{+}=\hbar \partial_{t}
$$

Here, the subscript $(2,1)$ means that $m=2$ and $j=1$.

- base order ' 0 ':

$$
\tilde{\Lambda}_{(2,0)}^{+}=\tilde{\Lambda}_{(2,1)}^{+}+\frac{1}{2 \hbar} O p\left(q_{O_{a}}\right)-\frac{1}{2 c \hbar} O p\left(q_{O_{b}}\right)
$$

- precision order '-1':

$$
\widetilde{\Lambda}_{(2,-1)}^{+}=\widetilde{\Lambda}_{(2,0)}^{+}-\frac{1}{2 \hbar} O p\left(q_{O_{z}}\right) \partial_{t}^{-1}
$$

- and lastly, precision order '-2':

$$
\widetilde{\Lambda}_{(2,-2)}^{+}=\widetilde{\Lambda}_{(2,-1)}^{+}+\frac{1}{4 \hbar}\left(\frac{1}{\hbar} O p\left(q_{O_{a}}\right) O p\left(q_{O_{z}}\right)-\frac{1}{\hbar} O p\left(\partial_{x} q_{O_{z}}\right)+O p\left(\partial_{t} q_{O_{z}}\right)\right) \partial_{t}^{-2}
$$

The results of these numerical simulations are shown by the contour plot of the logarithmic solution, $\log (|u(x, t)|)$, in the domain $\Omega$ under the effect of a time dependent electric field $A(t)=A_{0} \cos (\omega t)$ and potential $\Phi(t)=0.1 \sin (\omega t)$. Here $A_{0}=1$ and the following numerical data are used $\Delta t=\Delta x / c, \Delta x=20 / 500$. The solutions are presented by figures (5-1: a-d) and each figure compares two boundary conditions, the more accurate one on top is the boundary condition at $x_{r}=10$, while the bottom one is the less accurate boundary condition at $x_{l}=-10$. In the first figure (5-1: a), for example, we compare the leading order $\left(\partial_{x}+\widetilde{\Lambda}_{2,1}^{+}\right) u=\left(\partial_{x}+\hbar \partial_{t}\right) u=0$ to no condition at all. As we can see, each condition improves upon the previous one, and 


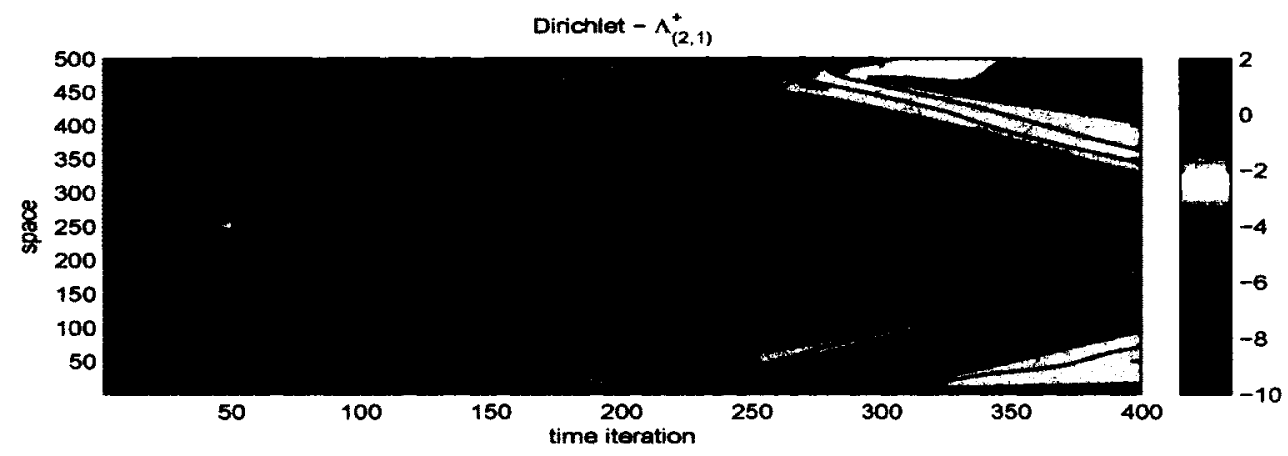

(a) $\widetilde{\Lambda}_{(2,1)}^{+}$(top) compared to Dirichlet(bot)

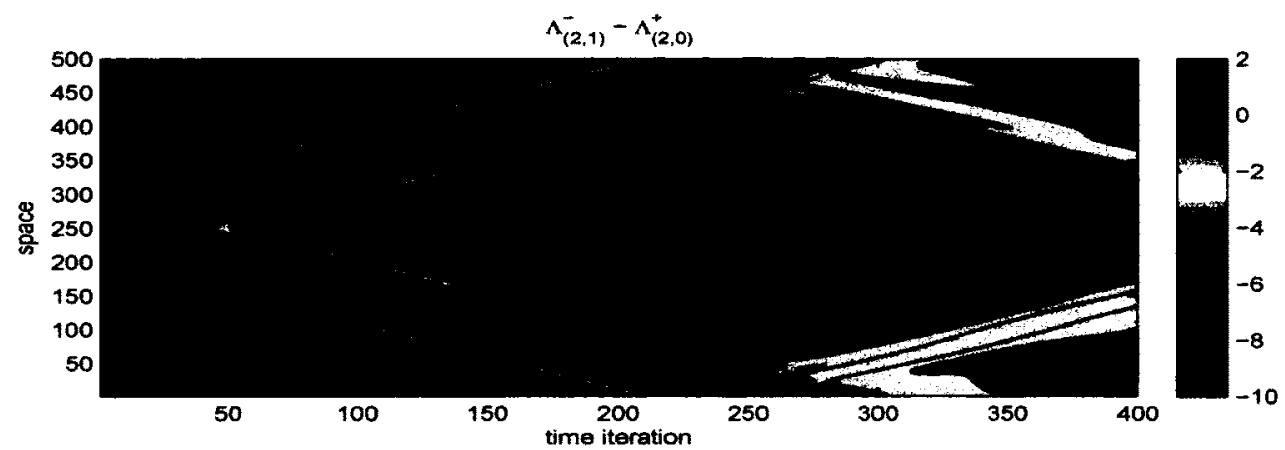

(b) $\widetilde{\Lambda}_{(2,0)}^{+}\left(\right.$top) compared to $\widetilde{\Lambda}_{(2,1)}^{-}($bot $)$

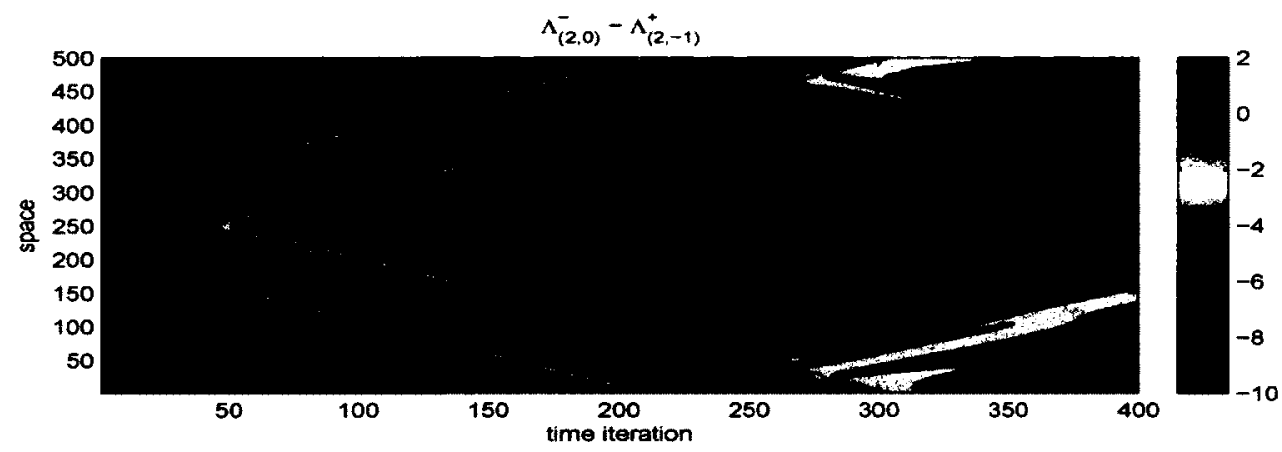

(c) $\widetilde{\Lambda}_{(2,-1)}^{+}\left(\right.$top) compared to $\tilde{\Lambda}_{(2,0)}^{-}($bot $)$

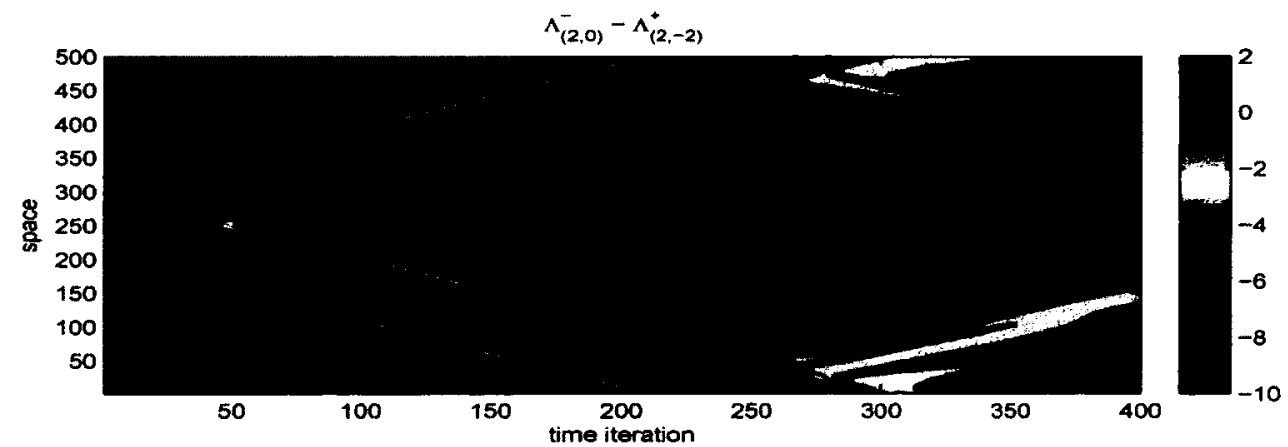

(d) $\tilde{\Lambda}_{(2,-2)}^{+}\left(\right.$top) compared to $\tilde{\Lambda}_{(2,0)}^{-}($bot $)$

Figure 5-1: Contour plot of $\log (|u(x, t)|)$ in $\Omega$ for different ABCs 
although the improvement between $\widetilde{\Lambda}_{(2,-1)}^{+}$and $\widetilde{\Lambda}_{(2,-2)}^{+}$is minimal, it is to be expected with a 'low order' interior discretization. 


\section{Chapter 6}

\section{Conclusion and future}

\section{considerations}

In this thesis, we constructed absorbing boundary conditions (ABC) for the 1-D and 2-D Klein-Gordon (KG) equation under the influence of time and space dependent electrodynamics potentials, which are relevant for problems in particle physics with high intensity lasers. We presented a small overview of a very large field of differential operators and we used their symbols to construct accurate and efficient ABCs. It is worth noting that the flexibility of the method introduced by Antoine \& Besse can be used to determine solutions to a wide range of partial differential equations, linear and non-linear, not necessarily limited to the one treated in this thesis and $[5,6]$. In the 1-D case, these ABCs would need to be compared to existing literature such as [18]. However, the 2-D ABCs are, as far as we know, new results in the literature and are worth investigating further. Nevertheless, several questions remain open,

From an analytical point of view: 
- The well-posedness of KG mixed problem,

$$
\begin{cases}P u=0 & \text { on } \Omega \times[0, T], \\ B u=0 & \text { on } \partial \Omega \times[0, T], \\ u(\cdot, 0)=\varphi_{0} & \text { on } \Omega\end{cases}
$$

and we cite [8] for the well-posedness of similar problems applied to Maxwell.

From a computational point of view:

- Construction of accurate and efficient discretization schemes for the non-local operators in time and space, such as the Riemann-Liouville operators [17]. The non-locality of these operators complicate the approximation and the study of convergence, as well as increase computational time and storage considerably.

- The discretization and testing of the $\mathrm{ABCs}$ for the 2-D KG equation is another important question as the conditions are new to the literature. One would possibly consider a finite element approach, however, only the space variable is local, and hence the non-locality of the other two variables takes us back to the previous point of properly handling the Riemann-Liouville integral.

Researching these questions would improve results, and allow a better application in practical problems, hence a deeper study in methods of discretization can be interesting subjects for future research considerations. 


\section{Bibliography}

[1] S. Alinhac and P. Gérard. Pseudo-differential Operators and the Nash-Moser Theorem (Graduate Studies in Mathematics). American Mathematical Society, english ed edition, 52007.

[2] X. Antoine, A. Arnold, C. Besse, M. Ehrhardt, and A. Schädle. A review of transparent and artificial boundary conditions techniques for linear and nonlinear Schrödinger equations. Communications in Computational Physics, 2008.

[3] X. Antoine, H. Barucq, and A. Bendali. Bayliss-Turkel-like radiation conditions on surfaces of arbitrary shape. Journal of Mathematical Analysis and Applications, 229(1):184-211, 1999.

[4] X. Antoine and C. Besse. Construction, structure and asymptotic approximations of a microdifferential transparent boundary condition for the linear Schrödinger equation. Journal des Mathematiques Pures et Appliquees, 80(7):701-738, 2001.

[5] X. Antoine, C. Besse, and S. Descombes. Artificial boundary conditions for onedimensional cubic nonlinear Schrödinger equations. SIAM Journal on Numerical Analysis, 43(6):pp. 2272-2293, 2006.

[6] X. Antoine, C. Besse, and P. Klein. Absorbing boundary conditions for the onedimensional Schrödinger equation with an exterior repulsive potential. Journal of Computational Physics, 228(2):312 - 335, 2009. 
[7] T. Aubin. A Course in Differential Geometry. Graduate Studies in Mathematics. American Mathematical Society, 2001.

[8] H. Barucq and M. Fontes. Well-posedness and exponential stability of Maxwelllike systems coupled with strongly absorbing layers. Journal de Mathématiques Pures et Appliquées, 87(3):253 - 273, 2007.

[9] B. Engquist and A. Majda. Absorbing boundary conditions for numerical simulation of waves. Proceedings of the National Academy of Sciences, 74(5):1765-1766, 1977.

[10] F. Fillion-Gourdeau, E. Lorin, and A. D. Bandrauk. Numerical solution of the time-dependent Dirac equation in coordinate space without fermion-doubling. Computer Physics Communications, 183:1403-1415, July 2012.

[11] L. Hörmander. Linear partial differential operators. Springer-Verlag, 1983.

[12] L. Hörmander. The analysis of linear partial differential operators I: distribution theory and Fourier analysis (Classics in Mathematics). Springer, 82003.

[13] L. Hörmander. The analysis of linear partial differential operators II: differential operators with constant coefficients (Classics in Mathematics). Springer, 122004.

[14] L. Hörmander. The analysis of linear partial differential operators III: pseudodifferential operators (Classics in Mathematics). Springer, 42007.

[15] L. Hörmander. The analysis of linear partial differential operators IV: Fourier integral operators (Classics in Mathematics). Springer, 52009.

[16] L. Nirenberg. Lectures on linear partial differential equations (CBMS Regional Conference Series in Mathematics No. 17). American Mathematical Society, 1973.

[17] M. Riesz. L'intégrale de Riemann-Liouville et le problème de Cauchy. Acta Mathematica, 81:1-222, 1949. 10.1007/BF02395016. 
[18] M. Ruf, H. Bauke, and C. H. Keitel. A real space split operator method for the Klein-Gordon equation. Journal of Computational Physics, 228(24):9092 - 9106, 2009.

[19] J. C. Strikwerda. Finite difference schemes and partial differential equations (Wadsworth and Brooks/Cole Mathematics Series). Springer, 1 edition, 91989. 